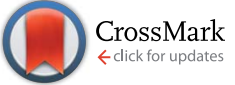

Cite this: RSC Adv., 2014, 4, 32148

\title{
Synthesis, characterization and gas permeation properties of anthracene maleimide-based polymers of intrinsic microporosity $\dagger$
}

\author{
Muntazim Munir Khan, ${ }^{a}$ Gisela Bengtson, ${ }^{a}$ Silvio Neumann, ${ }^{a}$ Md. Mushfequr Rahman, ${ }^{a}$ \\ Volker Abetz ${ }^{\text {ab }}$ and Volkan Filiz ${ }^{\star a}$
}

A series of new monomers containing dialkyl anthracene maleimide derivatives $[4 a, b(I-V)]$, which can be used as a precursor of a polymer of intrinsic microporosity (PIM) has been synthesized and characterized successfully. The homopolymers prepared via polycondensation with 2,3,5,6,tetrafluoroterephthalonitrile (TFTPN) and their copolymers in combination with 5,5',6,6'-tetrahydroxy$3,3,3^{\prime}, 3^{\prime}$-tetramethyl-1,1'-spirobisindane (TTSBI) were characterized by SEC, FT-IR, TGA, ${ }^{1} \mathrm{H}-\mathrm{NMR}, \mathrm{BET}$ surface area and gas transport properties. Compared to polymers derived from $4 \mathrm{a}(\mathrm{I}-\mathrm{V})$ monomers, the homopolymers and copolymers obtained from $4 \mathrm{~b}(\mathrm{I}-\mathrm{V})$ show improved solubility in common organic solvents and have high average molecular weight. Therefore they are able to form robust and transparent films. The gas transport properties of homopolymers and copolymers of $4 \mathrm{~b}(\mathrm{I}-\mathrm{V})$ show enhanced selectivity compared to PIM-1 for gas pairs such as $\mathrm{O}_{2} / \mathrm{N}_{2}, \mathrm{CO}_{2} / \mathrm{N}_{2}$ and $\mathrm{CO}_{2} / \mathrm{CH}_{4}$, followed by a slight decrease in permeability. The introduction of anthracene maleimide units (especially $4 \mathrm{blll}$ ) in the copolymer leads to more efficient chain packing and gives the copolymer a similar pore width distribution as PIM-1. As a consequence, the introduction of anthracene maleimide enhanced the $\mathrm{CO}_{2}$ selectivity of copolymers, compared to previously reported film forming polymers. Therefore, these polymers might be useful for gas separations relying on $\mathrm{CO}_{2}$ selectivity.

Received 22nd April 2014 Accepted 24th June 2014 DOI: $10.1039 / c 4 r a 03663 h$

www.rsc.org/advances

\section{Introduction}

During the past years, microporous organic materials gained increasing interest as they have a high potential in a variety of applications like gas separation or storage. These microporous polymer-based materials can be obtained by the extensive crosslinking of solvent swollen polymers (e.g., hypercrosslinked polystyrenes) ${ }^{1}$ or by preparing polymer networks using rigid monomers. ${ }^{2-4}$ In addition, certain highly rigid, non-crosslinked polymers, for example poly(trimethylsilylpropyne), ${ }^{5,6}$ certain polyimides, ${ }^{7}$ and a number of fluorinated polymers, ${ }^{8}$ form solids with very large free volumes so that they behaving similarly to microporous materials. However, only a very limited number of microporous and soluble polymers are known so far.

The most extensively studied purely organic materials with network and non-network structure, introduced by Budd et al., are termed as polymers of intrinsic microporosity (PIM), possessing good solubility in organic solvents, high thermal

${ }^{a}$ Helmholtz-Zentrum Geesthacht, Institute of Polymer Research, Max-Planck-Str. 1, 21502 Geesthacht, Germany.E-mail:volkan.filiz@hzg.demailto

${ }^{b}$ University of Hamburg, Institute of Physical Chemistry, Grindelallee 117, 20146 Hamburg, Germany

$\dagger$ Electronic supplementary information (ESI) available. See DOI: 10.1039/c4ra03663h stability and high surface area. The intrinsic microporosity is caused by a rigid and contorted molecular structure which cannot fill space efficiently, leaving molecular sizedinterconnected voids. ${ }^{9,10}$ This rigidity and contortion arises from the incorporation of non-linear 'sites of contortion' such as spirocyclic or triptycene units. ${ }^{11,12}$ Even compared with traditional widely used industrially microporous materials, such as zeolites or activated carbons. PIMs present a unique advantage, due to the combination of processability with a high degree of microporosity, which are attractive for applications such as adsorbents, gas separation and catalysis. Generally, PIMs can be easily prepared by polycondensation reaction (double aromatic nucleophilic substitution) using a combination of a tetrafunctional hydroxylated aromatic monomer and an activated tetrafluorinated or chlorinated aromatic monomer to form rigid dibenzodioxane linking groups resulting in ladder architecture. The first and most popular polymer of this type is referred to as PIM-1, derived by step-growth polymerization of 5,5',6,6'-tetrahydroxy-3,3,3, $3^{\prime} 3^{\prime}$-tetra-methylspirobisindane (TTSBI) and 2,3,5,6,-tetrafluoroterephthalonitrile (TFTPN) (Scheme 1). Despite the tremendous progress in the field of PIMs synthesis, there is still a need for the development of these materials that can exhibit higher (and more stable) free volume and which can be tailored for other applications such as gas separation, gas storage and liquid filtration. ${ }^{13}$ This can be 


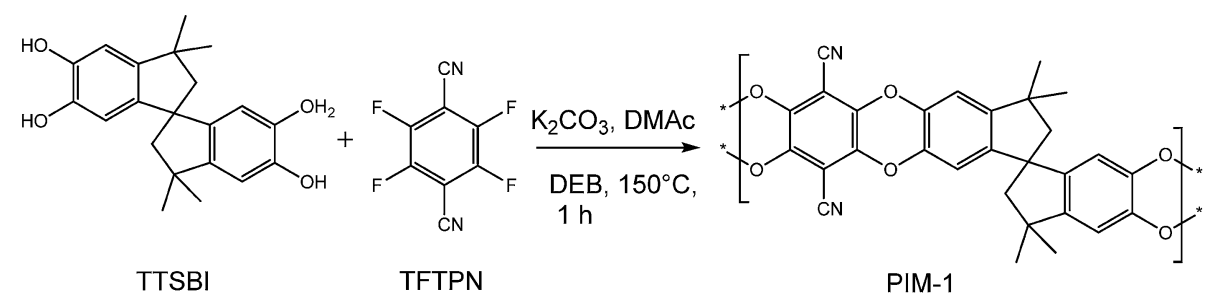

Scheme 1 Synthesis of PIM-1.

established via designing new monomers that will be utilized for polymer synthesis.

One of the structural components used previously for making PIM is 9,10-dimethyl-9,10-ethano-9,10-dihydro-2,3,6,7tetrahydroxy-anthracene (CO1)..$^{14,15}$ The resulting network polymer from the reaction between CO1 and TFTPN demonstrated an apparent BET surface area of $750 \mathrm{~m}^{2} \mathrm{~g}^{-1}$ with the microporosity presumably arising from the roof shaped of the CO1 units. ${ }^{14}$ Due to the potential for providing pre-formed concave binding sites of a fixed size and shape for specific adsorption, we are interested in synthesizing new examples of PIM with similar roof shaped components. Hence our attention was drawn to the anthracene maleimide unit, which has been exploited widely as a component of functional molecular systems including shape memory properties ${ }^{16}$ chemical and biological activities, ${ }^{17-19}$ click reactions ${ }^{20,21}$ and chiral auxiliaries. ${ }^{22}$ To our knowledge, there is no literature available so far on anthracene maleimide based polymers. In the context of PIM, it was anticipated that the roof shaped structure of the anthracene maleimide unit should ensure greater polymer rigidity as compared to $\mathrm{CO} 1$ and different substituents on the maleic anhydride ring could frustrate further the polymer chain packing which might enhance the intrinsic microporosity. In the present work, we describe synthesis, characterization and gas transport properties of new polymers of intrinsic microporosity (PIM) derived from dialkyl anthracene maleimide monomers, polycondensated with 2,3,5,6,tetrafluoroterephthalo-nitrile (TFTPN) as well as their copolymers in combination with tetrahydroxy monomer of PIM-1.

\section{Experimental}

\subsection{Materials and methods}

Most commercially available reagents were obtained from Sigma-Aldrich (Germany) and were used without further treat-

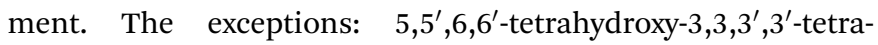
methyl-1,1'-spirobisindane (TTSBI, 97\%) was obtained from ABCR (Germany), and 2,3,5,6,-tetrafluoro-terephthalonitrile (TFTPN, 99\%) was kindly donated by Lanxess (Germany). TFTPN was sublimated twice in vacuum prior to use. Potassium carbonate $\left(\mathrm{K}_{2} \mathrm{CO}_{3},>99.5 \%\right)$ was dried overnight under vacuum at $120^{\circ} \mathrm{C}$ and milled in a ball mill for $15 \mathrm{~min}$. (Co)monomers $4 \mathrm{a} /$ b-I to $\mathbf{V}$ were prepared in our laboratory following the procedures described below. All reactions using air/moisture sensitive reagents were performed under argon atmosphere. NMR spectra were recorded on a Bruker AV300 NMR spectrometer operating at a field of 7 Tesla (300 MHz) using a $5 \mathrm{~mm}{ }^{1} \mathrm{H} /{ }^{13} \mathrm{C}$ TXI probe and a sample temperature of $298 \mathrm{~K} .{ }^{1} \mathrm{H}$ spectra were recorded applying a $10 \mathrm{~ms} 90^{\circ}$ pulse. ${ }^{13} \mathrm{C}$ spectra were recorded using dept-45 and dept-q135 sequences employing a waltz-16 decoupling scheme. The relaxation delay was chosen so that the sample was fully relaxed. FT-Infrared spectra were recorded on attenuated total reflectance (ATR-diamond crystal) mode with a Bruker ALPHA FT-IR spectrometer. The transmission measurements were done in a spectral range of $400-4000 \mathrm{~cm}^{-1}$ with a resolution of $4 \mathrm{~cm}^{-1}$ and average of 64 scans. Thermogravimetric analysis (TGA) onset temperature measurements were obtained using a Netzsch TG209 F1 Iris instrument under argon flow $\left(20 \mathrm{ml} \mathrm{min}{ }^{-1}\right)$ from $25^{\circ} \mathrm{C}$ to $900{ }^{\circ} \mathrm{C}$ at $10 \mathrm{~K} \mathrm{~min}^{-1}$. The retro Diels-Alder reaction temperature was investigated using thermogravimetric analysis coupled with FT-IR. Molar mass distributions were determined by Size Exclusion Chromatography (SEC) with chloroform as solvent using polystyrene calibrated standards. Low temperature $(77 \mathrm{~K})$ nitrogen $\left(\mathrm{N}_{2}\right)$ adsorption/desorption study of the polymer powder was undertaken using Rubotherm IsoSORP ${ }^{\circledR}$ instrument (Rubotherm GmbH, Bochum, Germany). Apparent surface area of the polymer was calculated from $\mathrm{N}_{2}$ adsorption data by multipoint BET analysis.

\subsection{Monomers synthesis}

2.2.1. Synthesis of 2,3,6,7-tetramethoxy-9,10-dimethylanthracene (1a). A mixture of 1,2-dimethoxybenzene and acetaldehyde (molar ratio $1: 1.7$ ) was added dropwise at $0-5{ }^{\circ} \mathrm{C}$ to a 3 -fold volume $\mathrm{H}_{2} \mathrm{SO}_{4}(70 \%)$ and stirred for $20 \mathrm{~min}$. The highly viscous lilac mixture was kept at room temperature for three days. The almost black mass was shovelled into ethanol-water $(1: 1)$, the resulting precipitate collected by filtration and dried in air. Two additional cleaning steps in chloroform and acetone give a light grey powder $(50-60 \%)$. Mp: $325{ }^{\circ} \mathrm{C} .{ }^{1} \mathrm{H}-\mathrm{NMR}(300 \mathrm{MHz}$, DMSOd6): $\delta(\mathrm{ppm}): 2.95(\mathrm{~s}, 6 \mathrm{H}), 4.08(\mathrm{~s}, 12 \mathrm{H}), 7.40(\mathrm{~s}, 4 \mathrm{H})$. FT-IR: 3019, 2996, 2963, 2833, 1633, 1495 (s), 1468, 1445, 1239, 1201, 1189, 1164 (s), 1013, 892, 828, $750 \mathrm{~cm}^{-1}$.

2.2.2. 2,3,6,7-Tetramethoxy-9,10-dibutylanthracene (1b). A cooled mixture of 1,2-dimethoxybenzene ( $0.1 \mathrm{~mol})$, pentanal $(0.1 \mathrm{~mol})$ and acetonitrile $(0.1 \mathrm{~mol})$ was added dropwise at $0-5{ }^{\circ} \mathrm{C}$ to stirred concentrated $\mathrm{H}_{2} \mathrm{SO}_{4}(50 \mathrm{ml})$ over a period of $1 \mathrm{~h}$. The reaction mixture was stirred at room temperature for $2 \mathrm{~h}$ and poured into ice-cold water. The precipitate was filtered and washed with copious amounts of water and washed with acetone to give a yellow powder (30\%), Mp: $218-220{ }^{\circ} \mathrm{C} .{ }^{1} \mathrm{H}-\mathrm{NMR}$ $\left(300 \mathrm{MHz}, \mathrm{CDCl}_{3}\right): \delta(\mathrm{ppm}): 1.06(\mathrm{t}, 6 \mathrm{H}), 1.61(\mathrm{~m}, 4 \mathrm{H})$, 
$1.81(\mathrm{~m}, 4 \mathrm{H}), 3.42(\mathrm{t}, 4 \mathrm{H}), 4.06(\mathrm{~s}, 12 \mathrm{H}), 6.84(\mathrm{~s}, 2 \mathrm{H}), 6.87(\mathrm{~s}, 2 \mathrm{H})$. ${ }^{13} \mathrm{C}-\mathrm{NMR}$ (75 MHz, $\mathrm{CDCl}_{3}$ ): $\delta$ (ppm): 14.2, 23, 28.3, 32.4, 55.6, 102.5, 124.7, 129.4, 148.7. FT-IR: 3006, 2949, 2960, 2828, 1633, 1553, 1434, 1239, 1199, 1189, 1164, 1013, 892, 828, $750 \mathrm{~cm}^{-1}$.

2.2.3. 2,3,6,7-Tetramethoxy-9,10-dimethyl-9,10,11,15-tetrahydro-9,10-[3,4]furanoanthracene-12,14-dione (2a). A mixture of $1 \mathrm{a}(13.64 \mathrm{~g}, 41.82 \mathrm{mmol})$ and maleic anhydride $(6.15 \mathrm{~g}, 62.73$ $\mathrm{mmol})$ in toluene $(600 \mathrm{ml})$ was refluxed at $120{ }^{\circ} \mathrm{C}$ for $2 \mathrm{~h}$. The solvent was removed from the grey suspension using short way distillation under reduced pressure to give a light grey solid (15.33 g, 86\%), Mp: $237{ }^{\circ} \mathrm{C} .{ }^{1} \mathrm{H}-\mathrm{NMR}$ (300 MHz, DMSO-d6): $\delta$ (ppm): 2.26 (s, 6H), 3.19 (s, 2H), 3.86 (s, 6H), 3.89 (s, 6H), 6.85 (s, 2H), 6.93 (s, 2H), FT-IR: 2951, 2829, 1821, 1763 (s), 1490, 1453, 1410, 1294, 1258, 1201, 1158, 1067, 1048, 1025, 930, $812 \mathrm{~cm}^{-1}$.

2.2.4. 2,3,6,7-Tetramethoxy-9,10-dibutyl-9,10,11,15-tetrahydro-9,10-[3,4]furanoanthracene-12,14-dione $(2 \mathrm{~b})$. $2 \mathrm{~b}$ was prepared by the same method as $\mathbf{2 a}$, using $\mathbf{1 b}$ instead of $\mathbf{1 a}$ to give a yellow solid; yield 90\%. Mp: $272{ }^{\circ} \mathrm{C} .{ }^{1} \mathrm{H}-\mathrm{NMR}(300 \mathrm{MHz}$, $\left.\mathrm{CDCl}_{3}\right): \delta(\mathrm{ppm}): 1.16(\mathrm{t}, 6 \mathrm{H}), 2.02(\mathrm{~m}, 4 \mathrm{H}), 2.43(\mathrm{t}, 4 \mathrm{H}), 2.9(\mathrm{~m}$, $4 \mathrm{H}), 3.55$ (s, 2H), $3.96(\mathrm{~s}, 12 \mathrm{H}), 6.84(\mathrm{~s}, 2 \mathrm{H}), 6.87(\mathrm{~s}, 2 \mathrm{H}) .{ }^{13} \mathrm{C}-$ NMR (75 MHz, $\mathrm{CDCl}_{3}$ ): $\delta$ (ppm): 14.3, 23.6, 27.1, 46.1, 48.4, 56.1, 106.5, 135, 146.7, 169.5. FT-IR: 2996, 2849, 1793, 1533, 1430, 1219, 1149, 1180, 1160, 1011, 889, $760 \mathrm{~cm}^{-1}$.

2.2.5. 2,3,6,7-Tetramethoxy-9,10-dimethyl-13-phenyl-9,10dihydro-9,10-[3,4] epipyrroloanthracene-12,14-dione (3a-I). $2 \mathrm{a}$ (3 $\mathrm{g}, 7.06 \mathrm{mmol})$ was added to a stirred solution of aniline $(3.49 \mathrm{ml}$, $38.16 \mathrm{mmol})$ and acetic acid $(13.5 \mathrm{ml})$. The reaction mixture was refluxed for $18 \mathrm{~h}$. After cooling acetic anhydride $(13.5 \mathrm{ml})$ was added. The dark brown reaction mixture was refluxed again at $150{ }^{\circ} \mathrm{C}$ for $6 \mathrm{~h}$, cooled and stirred at room temperature for $18 \mathrm{~h}$. The reaction mixture was poured in water-EtOH (2 lit), filtrated and washed with diethylether to give a beige solid (3.18 g, 90.3\%), Mp: $260{ }^{\circ} \mathrm{C} .{ }^{1} \mathrm{H}$ NMR (300 MHz, DMSO-d6): $\delta$ (ppm): 2.29 (s, 6H), 2.97 (s, 2H), $3.84(\mathrm{~s}, 6 \mathrm{H}), 3.89$ (s, 6H), 6.56/6.58 (d, $2 \mathrm{H}), 6.89(\mathrm{~s}, 2 \mathrm{H}), 6.98(\mathrm{~s}, 2 \mathrm{H}), 7.28$ (m, 3H), FT-IR: 2934, $1703(\mathrm{~s})$, 1607, 1492, 1460, 1405, 1374, 1282, 1256, 1231, 1195, 1165, 1036, 885, 867, 793, 756, $617 \mathrm{~cm}^{-1}$.

2.2.6. 2,3,6,7-Tetramethoxy-9,10-dibutyl-13-phenyl-9,10dihydro-9,10-[3,4] epipyrroloanthracene-12,14-dione (3b-I). 3b-I was prepared identically to $3 \mathbf{a}-\mathbf{I}$, using $\mathbf{2 b}$ instead of $\mathbf{2 a}$ to give a yellow solid, recrystallized from EtAc to give the product as white crystals (80\%), Mp: $271-275{ }^{\circ} \mathrm{C} .{ }^{1} \mathrm{H}$ NMR $\left(300 \mathrm{MHz}, \mathrm{CDCl}_{3}\right): \delta(\mathrm{ppm})$ : $1.06(\mathrm{t}, 6 \mathrm{H}), 1.85(\mathrm{~m}, 4 \mathrm{H}), 2.11(\mathrm{t}, 4 \mathrm{H}), 2.59(\mathrm{~m}, 4 \mathrm{H}), 3.31(\mathrm{~s}, 2 \mathrm{H}), 3.91$ (s, 12H), 6.55 (s, 2H), 6.71 (s, 2H), 7.29 (m, 3H). FT-IR: 2991, 2850, 1753, 1530, 1437, 1280, 1147, 1149, 1159, 1018, 880, $765 \mathrm{~cm}^{-1}$.

2.2.7. 2,3,6,7-Tetramethoxy-9,10-dimethyl-13-(4-methylphenyl)-9,10-dihydro-9,10-[3,4] epipyrroloanthracene-12,14dione (3a-II). 3a-II was prepared by the same method as 3a-I, using toluidine instead of aniline and resulted in a light brown solid (80\%). Mp: 258-261 ${ }^{\circ} \mathrm{C} .{ }^{1} \mathrm{H}$ NMR (300 MHz, DMSO-d6): $\delta$ (ppm): 2.29 (s, 3H), 2.30 (s, 6H), 3.03 (s, 2H), 3.85 (s, 6H), 3.91 (s, $6 \mathrm{H}), 6.45$ (d, 2H), 6.89 (s, 2H), 6.99 (s, 2H), 7.12 (d, 2H), FT-IR: 1701, 1513, 1494, 1449, 1404, 1195, 1165, 1039 (s), 816, 794, $783 \mathrm{~cm}^{-1}$.

2.2.8. 2,3,6,7-Tetramethoxy-9,10-dibutyl-13-(4-methylphenyl)9,10-dihydro-9,10-[3,4] epipyrroloanthracene-12,14-dione (3bII). 3b-II was prepared using $\mathbf{2 b}$ and 4-methylaniline. Recrystallization from EtAc gave the product as white crystals (40\%),
Mp: $276-278{ }^{\circ} \mathrm{C} .{ }^{1} \mathrm{H}$ NMR (300 MHz, $\left.\mathrm{CDCl}_{3}\right): \delta(\mathrm{ppm}): 1.00(\mathrm{t}$, $6 \mathrm{H}), 1.66(\mathrm{~m}, 4 \mathrm{H}), 1.74(\mathrm{t}, 4 \mathrm{H}), 2.51(\mathrm{~m}, 7 \mathrm{H}), 3.11(\mathrm{~s}, 2 \mathrm{H}), 3.94(\mathrm{~s}$, $12 \mathrm{H}), 6.61$ (m, 6H), 7.71 (s, 2H). FT-IR: 2997, 2850, 1740, 1499, 1288, 1097, 1139, 1011, 885, $760 \mathrm{~cm}^{-1}$.

2.2.9. 2,3,6,7-Tetramethoxy-9,10-dimethyl-13-(2,4,6-trimethylphenyl)-9,10-dihydro-9,10-[3,4] epipyrroloanthracene-12,14-dione (3a-III). 3a-III was prepared by using 2,4,6-trimethylaniline to give light brown solid (97.3\%). Mp: $256{ }^{\circ} \mathrm{C} .{ }^{1} \mathrm{H}$ NMR $(300 \mathrm{MHz}$, DMSO-d6): $\delta$ (ppm): 1.04 (s, 3H), 1.91 (s, 3H), 2.21 (s, 3H), 2.32 (s, $6 \mathrm{H}), 3.12(\mathrm{~s}, 2 \mathrm{H}), 3.84(\mathrm{~s}, 6 \mathrm{H}), 3.90(\mathrm{~s}, 6 \mathrm{H}), 6.73(\mathrm{~s}, 1 \mathrm{H}), 6.85(\mathrm{~s}$, 1H), 6.90 (s, 2H), 6.96 (s, 2H), FT-IR: 2937, 1698 (s), 1490, 1466, $1376,1287,1201,1165,1043,866,826,788 \mathrm{~cm}^{-1}$.

2.2.10. 2,3,6,7-Tetramethoxy-9,10-dibutyl-13-(2,4,6-trimethylphenyl)-9,10-dihydro-9,10-[3,4] epipyrroloanthracene-12,14-dione (3b-III). 3b-III was prepared using $2 \mathbf{b}$ instead of $2 \mathbf{a}$ to give a yellow solid and recrystallized from EtAc to give desired product $(40 \%)$ as white crystals, Mp: $260-265{ }^{\circ} \mathrm{C} .{ }^{1} \mathrm{H}$ NMR $\left(300 \mathrm{MHz}, \mathrm{CDCl}_{3}\right): \delta$ (ppm): $1.01(\mathrm{~m}, 6 \mathrm{H}), 1.58(\mathrm{~m}, 4 \mathrm{H}), 1.95(\mathrm{~m}, 5 \mathrm{H}), 2.10(\mathrm{~m}, 5 \mathrm{H}), 2.59$ $(\mathrm{m}, 2 \mathrm{H}), 3.41(\mathrm{~s}, 2 \mathrm{H}), 3.95(\mathrm{~s}, 12 \mathrm{H}), 6.61(\mathrm{~s}, 2 \mathrm{H}), 6.71(\mathrm{~s}, 2 \mathrm{H}), 6.64$ (s, 2H), 6.81 (s, 2H). FT-IR: 2995, 2849, 1730, 1509, 1298, 1197, 1109, 1014, 881, $759 \mathrm{~cm}^{-1}$.

2.2.11. 2,3,6,7-Tetramethoxy-9,10-dimethyl-13-(4-(t-butyl) phenyl)-9,10-dihydro-9,10-[3,4] epipyrroloanthracene-12,14-dione (3a-IV). 3a-IV was prepared using 4-t-butylaniline instead of aniline to give a white solid (71\%). Mp: $270{ }^{\circ} \mathrm{C} .{ }^{1} \mathrm{H}$ NMR (300 MHz, DMSOd6): $\delta(\mathrm{ppm}): 1.26(\mathrm{~s}, 9 \mathrm{H}), 2.31(\mathrm{~s}, 6 \mathrm{H}), 3.03(\mathrm{~s}, 2 \mathrm{H}), 3.86(\mathrm{~s}, 6 \mathrm{H}), 3.91$ (s, 6H), 6.50 (d, 2H), 6.89 (s, 2H), 6.99 (s, 2H), 7.31 (d, 2H), FT-IR: 2937, 2841, 1771, 1509, 1284, 1198, 1109, 1014, 822, $784 \mathrm{~cm}^{-1}$.

2.2.12. 2,3,6,7-Tetramethoxy-9,10-dibutyl-13-(4-(t-butyl) phenyl)9,10-dihydro-9,10-[3,4] epipyrroloanthracene-12,14-dione (3b-IV). 3bIV was prepared like $\mathbf{3 a - I V}$ using $\mathbf{2} \mathbf{b}$ and $\mathbf{4} t$-butylaniline to give a yellow solid, recrystallized from EtAc as white crystals (60\%), Mp: $265-268{ }^{\circ} \mathrm{C} .{ }^{1} \mathrm{H}$ NMR (300 MHz, $\left.\mathrm{CDCl}_{3}\right): \delta(\mathrm{ppm}): 1.1(6 \mathrm{H})$, $1.2(9 \mathrm{H}), 1.6(\mathrm{~m}, 4 \mathrm{H}), 1.9(\mathrm{t}, 2 \mathrm{H}), 2.1(\mathrm{~m}, 2 \mathrm{H}), 2.6(\mathrm{~m}, 2 \mathrm{H}), 3.3(\mathrm{~s}$, $2 \mathrm{H}), 3.9(\mathrm{~s}, 12 \mathrm{H}), 4.1(\mathrm{~s}, 2 \mathrm{H}), 6.6(\mathrm{~s}, 2 \mathrm{H}), 6.9(\mathrm{~s}, 4 \mathrm{H}), 7.4(\mathrm{~s}, 2 \mathrm{H})$. FT-IR: 2991, 2850, 1710, 1519, 1288, 1189, 1110, 888, $759 \mathrm{~cm}^{-1}$.

2.2.13. 2,3,6,7-Tetramethoxy-9,10-dimethyl-13-(4-ethoxyphenyl)9,10-dihydro-9,10-[3,4] epipyrroloanthracene-12,14-dione (3a-V). 3a-V was prepared by using 4-ethoxyaniline to give a light brown solid (yield 76\%). Mp: $262{ }^{\circ} \mathrm{C} .{ }^{1} \mathrm{H}$ NMR (300 MHz, DMSO-d6): $\delta$ (ppm): 1.37 (t, 3H), $2.30(\mathrm{~s}, 6 \mathrm{H}), 3.01(\mathrm{~s}, 2 \mathrm{H}), 3.86(\mathrm{~s}, 6 \mathrm{H}), 3.91(\mathrm{~s}$, $6 \mathrm{H}), 3.96(\mathrm{q}, 2 \mathrm{H}), 6.46(\mathrm{~d}, 2 \mathrm{H}), 6.79(\mathrm{~d}, 2 \mathrm{H}), 6.89(\mathrm{~s}, 2 \mathrm{H}), 6.99(\mathrm{~s}$, 2H), FT-IR: 1699, 1509, 1493, 1439, 1385, 1284, 1243, 1195, 1163 (s), $1037(\mathrm{~s}), 785,761 \mathrm{~cm}^{-1}$.

2.2.14. 2,3,6,7-Tetramethoxy-9,10-dibutyl-13-(4-ethoxyphenyl)9,10-dihydro-9,10-[3,4] epipyrroloanthracene-12,14-dione (3b-V). $\mathbf{3 b}-\mathbf{V}$ was prepared by reaction of $\mathbf{2 b}$ with 4 -ethoxyaniline to give a yellow solid and recrystallized from EtAc to give desired product as white crystals (yield 64\%), Mp: $285-288{ }^{\circ} \mathrm{C} .{ }^{1} \mathrm{H}$ NMR $(300 \mathrm{MHz}$, $\left.\mathrm{CDCl}_{3}\right): \delta(\mathrm{ppm}): 1.11(\mathrm{t}, 6 \mathrm{H}), 1.41(\mathrm{t}, 3 \mathrm{H}), 1.72(\mathrm{~m}, 4 \mathrm{H}), 1.8(\mathrm{t}, 4 \mathrm{H})$, $2.10(\mathrm{~m}, 2 \mathrm{H}), 2.6(\mathrm{~m}, 2 \mathrm{H}), 3.3(\mathrm{~s}, 2 \mathrm{H}), 3.9(\mathrm{~s}, 12 \mathrm{H}), 4.0(\mathrm{t}, 2 \mathrm{H}), 6.4(\mathrm{~s}$, 2H), 6.6 (s, 2H), 6.7 (s, 2H), 6.8 (s, 2H). FT-IR: 3005, 2941, 2730, 1703, 1510, 1288, 1097, 1014, 799, $759 \mathrm{~cm}^{-1}$.

2.2.15. 2,3,6,7-Tetrahydroxy-9,10-dimethyl-13-phenyl-9,10dihydro-9,10-[3,4] epipyrroloanthracene-12,14-dione (4a-I)

General procedure for demethylation. 3a-I (3.34 g, $6.68 \mathrm{mmol})$ was dissolved in $20 \mathrm{ml}$ dichloromethane (DCM) and added 
slowly with cooling in an ice-bath to boron tribromide $(1.26 \mathrm{ml}$, $13.37 \mathrm{mmol}$ ), dissolved in $40 \mathrm{ml}$ DCM under argon atmosphere. The reaction mixture was stirred at room temperature for $18 \mathrm{~h}$ and then poured to water ( 1 litre). The acidic $\mathrm{pH}(=5)$ was neutralized with $1 \% \mathrm{NaOH}$ till $\mathrm{pH}=7-8$. The precipitate was filtered and washed with copious amount of water, dried in vacuum at $60{ }^{\circ} \mathrm{C}$ to give a grey solid $(2.68 \mathrm{~g}, 90.7 \%), \mathrm{Mp}:>300{ }^{\circ} \mathrm{C}$. ${ }^{1} \mathrm{H}$ NMR (300 MHz, DMSO-d6): $\delta(\mathrm{ppm}): 2.00$ (s, 6H), 2.97 (s, 2H), $6.52(\mathrm{dd}, 2 \mathrm{H}), 6.55(\mathrm{~s}, 2 \mathrm{H}), 6.65(\mathrm{~s}, 2 \mathrm{H}), 7.33(\mathrm{~m}, 3 \mathrm{H}), 8.73(\mathrm{~s}, 4 \mathrm{H})$, ${ }^{13} \mathrm{C}$ NMR (75 MHz, DMSO-d6): $\delta$ (ppm): 16.1, 42.6, 53.0, 110.1, $110.4,127.1$, 128.7, 129.1, 132.5, 133.3, 136.7, 143.0, 143.3, 175.7. FT-IR: 3348, 2910, 1768, 1691, 1594, 1493, 1445, 1392, 1310 (s), 1236, 1210, 1184, 1153, 998, 757, 709, 691, $622 \mathrm{~cm}^{-1}$. $\mathrm{C}_{26} \mathrm{H}_{21} \mathrm{NO}_{6}$ calculated: $\mathrm{C}=70.41 \%, \mathrm{H}=4.78 \%, \mathrm{~N}=3.16 \%, \mathrm{O}=$ $21.66 \%$, found: $\mathrm{C}=68.37 \%, \mathrm{H}=5.02 \%, \mathrm{~N}=3.15 \%, \mathrm{O}=$ $22.86 \%$. TGA $_{\text {(onset) }}=312{ }^{\circ} \mathrm{C}$.

2.2.16. 2,3,6,7-Tetrahydroxy-9,10-dibutyl-13-phenyl-9,10dihydro-9,10-[3,4] epipyrroloanthracene-12,14-dione (4b-I). 4b-I was prepared from 3b-I, as a grey powder (53\%), ${ }^{1} \mathrm{H}$ NMR (300 MHz, $\left.\mathrm{CDCl}_{3}\right): \delta(\mathrm{ppm}): 1.05(\mathrm{t}, 6 \mathrm{H}), 1.89(\mathrm{~m}, 4 \mathrm{H}), 2.14(\mathrm{t}, 4 \mathrm{H})$, $2.63(\mathrm{~m}, 4 \mathrm{H}), 3.35(\mathrm{~s}, 2 \mathrm{H}), 6.51(\mathrm{~d}, 2 \mathrm{H}), 6.65(\mathrm{~s}, 2 \mathrm{H}) .6 .73(\mathrm{~s}, 2 \mathrm{H})$, $7.32(\mathrm{~m}, 3 \mathrm{H}), 8.67$ (s, 2H), $8.72(\mathrm{~s}, 2 \mathrm{H}) .{ }^{13} \mathrm{C} \mathrm{NMR}\left(75 \mathrm{MHz}, \mathrm{CDCl}_{3}\right)$ : $\delta$ (ppm): 14.3, 24.2, 26.6, 41.8, 47.9, 48.9, 100.8, 127.8, 128.5, 129.4, 132.4, 135.9, 151.2, 171.1. FT-IR: 3301, 3005, 2801, 1790, 1509, 1310, 1201, 1041, $950 \mathrm{~cm}^{-1} \cdot \mathrm{C}_{32} \mathrm{H}_{33} \mathrm{NO}_{6}$, calculated: $\mathrm{C}=$ $72.83 \%, \mathrm{H}=6.31 \%, \mathrm{~N}=2.66 \%, \mathrm{O}=18.21 \%$, found: $\mathrm{C}=69.94 \%$, $\mathrm{H}=6.51 \%, \mathrm{~N}=2.31 \%, \mathrm{O}=18.06 \%$. $\mathrm{TGA}_{\text {(onset) }}=316^{\circ} \mathrm{C}$.

2.2.17. 2,3,6,7-Tetrahydroxy-9,10-dimethyl-13-(4-methylphenyl)9,10-dihydro-9,10-[3,4] epipyrroloanthracene-12,14-dione (4a-II). 4a-II, a light brown solid (83.5\%), ${ }^{1} \mathrm{H}$ NMR (300 MHz, DMSO-d6): $\delta$ (ppm): 1.98 (s, 6H), 2.27 (s, 3H), 2.95 (s, 2H), 6.40 (d, 2H), 6.63 (s, 2H), 6.77 (s, 2H), 7.13 (d, 2H), 8.75 (br, 4H). ${ }^{13} \mathrm{C}$ NMR (75 MHz, DMSO-d6): $\delta$ (ppm): 16.2, 21.0, 43.0, 52.6, 110.2, 110.4, 126.9, 129.6, 133.4 , 136.7, 138.3, 142.9, 143.3, 175.6. FT-IR: 3484, 1684 (s), 1512, 1494, 1448, 1401, 1299 (s), 1210, 877, 819, $806 \mathrm{~cm}^{-1}$. $\mathrm{C}_{27} \mathrm{H}_{23} \mathrm{NO}_{6}$, calculated: $\mathrm{C}=70.87 \%, \mathrm{H}=5.07 \%, \mathrm{~N}=3.06 \%, \mathrm{O}=$ $20.99 \%$, found: $\mathrm{C}=68.46 \%, \mathrm{H}=5.05 \%, \mathrm{~N}=3.01 \%, \mathrm{O}=$ $21.98 \%$. TGA $_{\text {(onset) }}=316^{\circ} \mathrm{C}$.

2.2.18. 2,3,6,7-Tetrahydroxy-9,10-dibutyl-13-(4-methylphenyl)9,10-dihydro-9,10-[3,4] epipyrroloanthracene-12,14-dione (4b-II). 4b-II, a brown powder (50\%), ${ }^{1} \mathrm{H}$ NMR $\left(300 \mathrm{MHz}, \mathrm{CDCl}_{3}\right): \delta(\mathrm{ppm})$ : $1.05(\mathrm{t}, 6 \mathrm{H}), 1.61(\mathrm{~m}, 4 \mathrm{H}), 1.77(\mathrm{t}, 4 \mathrm{H}), 2.50(\mathrm{~m}, 7 \mathrm{H}), 3.13(\mathrm{~s}, 2 \mathrm{H})$, $6.64(\mathrm{~m}, 6 \mathrm{H}), 7.78(\mathrm{~s}, 2 \mathrm{H}), 8.60(\mathrm{~s}, 2 \mathrm{H}), 8.72(\mathrm{~s}, 2 \mathrm{H}) .{ }^{13} \mathrm{C}$ NMR (75 $\left.\mathrm{MHz}, \mathrm{CDCl}_{3}\right): \delta$ (ppm): 14.5, 21.01, 23.53, 27, 28.2, 47.7, 110.5, 111.4, 126.8, 129.5. FT-IR: 3351, 3010, 1709, 1449, 1320, 1211, 1044, $880 \mathrm{~cm}^{-1} \cdot \mathrm{C}_{33} \mathrm{H}_{35} \mathrm{NO}_{6}$, calculated: $\mathrm{C}=73.16 \%, \mathrm{H}=6.52 \%, \mathrm{~N}=$ $2.59 \%, \mathrm{O}=17.73 \%$, found: $\mathrm{C}=73.10 \%, \mathrm{H}=6.54 \%, \mathrm{~N}=2.83 \%$, $\mathrm{O}=17.54 \%$. $\mathrm{TGA}_{\text {(onset) }}=312{ }^{\circ} \mathrm{C}$.

2.2.19. 2,3,6,7-Tetrahydroxy-9,10-dimethyl-13-(2,4,6-trimethylphenyl)-9,10-dihydro-9,10-[3,4] epipyrroloanthracene-12,14-dione (4a-III). 4a-III, a light brown solid (91.5\%), ${ }^{1} \mathrm{H}$ NMR $(300 \mathrm{MHz}$, DMSO-d6): $\delta$ (ppm): 0.99 (s, 3H), 1.83 (s, 3H), 2.00 (s, 6H), 2.18 (s, $3 \mathrm{H}), 3.12(\mathrm{~s}, 2 \mathrm{H}), 6.65(\mathrm{~s}, 2 \mathrm{H}), 6.75(\mathrm{~s}, 2 \mathrm{H}), 6.78(\mathrm{~s}, 1 \mathrm{H}), 6.86(\mathrm{~s}$, $1 \mathrm{H}), 8.67 / 8.69$ (2s, $4 \mathrm{H}) .{ }^{13} \mathrm{C}$ NMR (75 MHz, DMSO-d6): $\delta$ (ppm): 16.0, 16.3, 17.5, 20.9, 42.6, 52.6, 110.7, 109.9, 128.7, 128.4, 133.7, 135.2, 135.7, 137.3, 138.4, 142.8, 143.7, 175.6. FT-IR: 3314, 1687 (s), 1451, 1383, 1303 (s), 1207, 1188, 1159, $880 \mathrm{~cm}^{-1} \cdot \mathrm{C}_{29} \mathrm{H}_{27} \mathrm{NO}_{6}$, calculated: $\mathrm{C}=71.73 \%, \mathrm{H}=5.61 \%, \mathrm{~N}=2.89 \%, \mathrm{O}=19.78 \%$, found: $\mathrm{C}=69.72 \%, \mathrm{H}=5.78 \%, \mathrm{~N}=3.10 \%, \mathrm{O}=20.58 \%$. $\mathrm{TGA}_{(\text {onset })}=316^{\circ} \mathrm{C}$.

2.2.20. 2,3,6,7-Tetrahydroxy-9,10-dibutyl-13-(2,4,6-trimethylphenyl)-9,10-dihydro-9,10-[3,4] epipyrroloanthracene-12,14-dione (4b-III). 4b-III, a light brown powder (60\%), ${ }^{1} \mathrm{H}$ NMR $(300 \mathrm{MHz}$, $\left.\mathrm{CDCl}_{3}\right): \delta(\mathrm{ppm}): 1.01(\mathrm{~s}, 3 \mathrm{H}), 1.08(\mathrm{~m}, 6 \mathrm{H}), 1.63(\mathrm{~m}, 4 \mathrm{H}), 1.94(\mathrm{~m}$, $5 \mathrm{H}), 2.12(\mathrm{~m}, 5 \mathrm{H}), 2.64(\mathrm{~m}, 2 \mathrm{H}), 3.46(\mathrm{~s}, 2 \mathrm{H}), 6.64(\mathrm{~s}, 2 \mathrm{H}), 6.69(\mathrm{~s}$, $2 \mathrm{H}), 6.77(\mathrm{~s}, 2 \mathrm{H}), 6.86(\mathrm{~s}, 2 \mathrm{H}), 8.60(\mathrm{~s}, 2 \mathrm{H}), 8.70(\mathrm{~s}, 2 \mathrm{H}) .{ }^{13} \mathrm{C} \mathrm{NMR}$ (75 $\mathrm{MHz}, \mathrm{CDCl}_{3}$ ): $\delta$ (ppm): 14.6, 15.6, 17.6, 20.9, 23.6, 27.1, 28.6, 47.3, 110.9, 111.3, 128.7, 128.8. FT-IR: 3410, 2910, 1711, 1355, $1100,800 \mathrm{~cm}^{-1} . \mathrm{C}_{35} \mathrm{H}_{39} \mathrm{NO}_{6}$, calculated: $\mathrm{C}=73.78 \%, \mathrm{H}=6.9 \%$, $\mathrm{N}=2.46 \%, \mathrm{O}=16.86 \%$, found: $\mathrm{C}=73.75 \%, \mathrm{H}=6.90 \%, \mathrm{~N}=$ $2.44 \%, \mathrm{O}=16.91 \%$. $\mathrm{TGA}_{\text {(onset) }}=316^{\circ} \mathrm{C}$.

2.2.21. 2,3,6,7-Tetrahydroxy-9,10-dimethyl-13-(4-(t-butyl) phenyl)-9,10-dihydro-9,10-[3,4] epipyrroloanthracene-12,14-dione (4a-IV). 4a-IV, a light brown solid (80\%), ${ }^{1} \mathrm{H}$ NMR $(300 \mathrm{MHz}$, DMSO-d6): $\delta$ (ppm): 1.24 (s, 9H), 1.98 (s, 6H), 2.96 (s, 2H), 6.43 (d, $2 \mathrm{H}), 6.64$ (s, 2H), 6.77 (s, 2H), 7.33 (d, 2H), 8.76 (br, 4H). ${ }^{13} \mathrm{C}$ NMR (75 MHz, DMSO-d6): $\delta$ (ppm): 16.2, 31.4, 34.8, 43.1, 52.9, 110.4, 110.2, 125.9, 126.7, 129.9, 133.3, 142.9, 143.3, 151.2, 175.7. FT-IR: 3501, 3330, 1689, 1449, 1301 (s), 1219, 1144, $884 \mathrm{~cm}^{-1}$. $\mathrm{C}_{30} \mathrm{H}_{29} \mathrm{NO}_{6}$, calculated: $\mathrm{C}=72.13 \%, \mathrm{H}=5.85 \%, \mathrm{~N}=2.8 \%, \mathrm{O}=$ $17.22 \%$, found: $\mathrm{C}=71.28 \%, \mathrm{H}=6.07 \%, \mathrm{~N}=2.54 \%, \mathrm{O}=19.2 \%$. $\mathrm{TGA}_{(\text {onset })}=295{ }^{\circ} \mathrm{C}$.

2.2.22. 2,3,6,7-Tetrahydroxy-9,10-dibutyl-13-(4-(t-butyl) phenyl)9,10-dihydro-9,10-[3,4] epipyrroloanthracene-12,14-dione (4b-IV). 4bIV, a white powder (75\%), ${ }^{1} \mathrm{H}$ NMR $\left(300 \mathrm{MHz}, \mathrm{CDCl}_{3}\right) \delta$ (ppm): 1.1 (6H), $1.2(9 \mathrm{H}), 1.6(\mathrm{~m}, 4 \mathrm{H}), 1.9(\mathrm{t}, 4 \mathrm{H}), 2.1(\mathrm{~m}, 2 \mathrm{H}), 2.6(\mathrm{~m}, 2 \mathrm{H}), 3.3(\mathrm{~s}$, $2 \mathrm{H}), 6.4(\mathrm{~s}, 2 \mathrm{H}), 6.6(\mathrm{~s}, 2 \mathrm{H}), 6.7(\mathrm{~s}, 2 \mathrm{H}), 7.3(\mathrm{~s}, 2 \mathrm{H}), 8.7(\mathrm{~s}, 4 \mathrm{H}) .{ }^{13} \mathrm{C}$ NMR (75 MHz, $\mathrm{CDCl}_{3}$ ): $\delta$ (ppm): 14.6, 15.5, 23.5, 27, 28.3, 47.7, 55.5, 65.3, 102.5, 108.8, 110.5, 111.4, 125.9, 126.6. FT-IR: 3400, 3010, 1710, 1350, 1100, $820 \mathrm{~cm}^{-1} \cdot \mathrm{C}_{36} \mathrm{H}_{41} \mathrm{NO}_{6}$, calculated: $\mathrm{C}=74.06 \%, \mathrm{H}=$ $7.08 \%, \mathrm{~N}=2.4 \%, \mathrm{O}=16.45 \%$, found: $\mathrm{C}=73.22 \%, \mathrm{H}=7.15 \%, \mathrm{~N}=$ $2.36 \%, \mathrm{O}=17.16 \%$. $\mathrm{TGA}_{\text {(onset) }}=317^{\circ} \mathrm{C}$.

2.2.23. 2,3,6,7-Tetrahydroxy-9,10-dimethyl-13-(4-ethoxyphenyl)9,10-dihydro-9,10-[3,4] epipyrroloanthracene-12,14-dione (4a-V). 4a$\mathbf{V}$, a light brown solid (84\%), ${ }^{1} \mathrm{H}$ NMR (300 MHz, DMSO-d6): $\delta$ (ppm): 1.29 (t, 3H), $1.98(\mathrm{~s}, 6 \mathrm{H}), 2.94$ (s, 2H), 3.99 (q, 2H), 6.42 (d, $2 \mathrm{H}), 6.64(\mathrm{~s}, 2 \mathrm{H}), 6.77(\mathrm{~s}, 2 \mathrm{H}), 6.86(\mathrm{~d}, 2 \mathrm{H}), 8.71(\mathrm{~s}, 4 \mathrm{H}),{ }^{13} \mathrm{C}$ NMR (75 MHz, DMSO-d6): $\delta$ (ppm): 14.9, 16.1, 43.0, 52.8, 63.7, 110.2, $110.4,114.8,124.9,133.4,136.7,142.9,143.2$, 158.5, 175.7. FTIR: 3347, 2980, 2936, 1757, 1512, 1298, 1164, $879 \mathrm{~cm}^{-1}$. $\mathrm{C}_{28} \mathrm{H}_{25} \mathrm{NO}_{7}$, calculated: $\mathrm{C}=68.99 \%, \mathrm{H}=5.17 \%, \mathrm{~N}=2.87 \%, \mathrm{O}=$ $22.97 \%$, found: $\mathrm{C}=67.45 \%, \mathrm{H}=5.12 \%, \mathrm{~N}=2.65 \%, \mathrm{O}=$ $24.25 \%$. TGA $_{(\text {onset })}=317^{\circ} \mathrm{C}$.

2.2.24. 2,3,6,7-Tetrahydroxy-9,10-dibutyl-13-(4-ethoxyphenyl)9,10-dihydro-9,10-[3,4] epipyrroloanthracene-12,14-dione (4b-V). 4b-V, a white powder (70\%), ${ }^{1} \mathrm{H}$ NMR $\left(300 \mathrm{MHz}, \mathrm{CDCl}_{3}\right): \delta(\mathrm{ppm})$ : $1.1(\mathrm{t}, 6 \mathrm{H}), 1.31(\mathrm{t}, 3 \mathrm{H}), 1.72(\mathrm{~m}, 4 \mathrm{H}), 1.8(\mathrm{t}, 4 \mathrm{H}), 2.10(\mathrm{~m}, 2 \mathrm{H}), 2.6$ $(\mathrm{m}, 2 \mathrm{H}) 3.3(\mathrm{~s}, 2 \mathrm{H}), 4.0(\mathrm{t}, 2 \mathrm{H}), 6.4(\mathrm{~s}, 2 \mathrm{H}), 6.6(\mathrm{~s}, 2 \mathrm{H}), 6.7(\mathrm{~s}, 2 \mathrm{H}), 6.8$ (s, 2H), $8.60(\mathrm{~s}, 2 \mathrm{H}), 8.72$ (s, 2H). ${ }^{13} \mathrm{C}$ NMR (75 MHz, DMSO-d6): $\delta$ (ppm):14.5, 23.4, 27, 28.3, 47.6, 56.3, 63.6, 108.6, 110.5, 111.4, 114.7, 115.6, 128.2. FT-IR: 3405, 3000, 1650, 1500, 1350, 1012, 850 $\mathrm{cm}^{-1} . \mathrm{C}_{34} \mathrm{H}_{37} \mathrm{NO}_{7}$, calculated: $\mathrm{C}=71.42 \%, \mathrm{H}=6.53 \%, \mathrm{~N}=2.45 \%$, $\mathrm{O}=19.6 \%$, found: $\mathrm{C}=69.33 \%, \mathrm{H}=6.86 \%, \mathrm{~N}=2.39 \%, \mathrm{O}=$ $20.85 \%$. $\mathrm{TGA}_{\text {(onset) }}=295^{\circ} \mathrm{C}$. 


\subsection{Polymer synthesis}

2.3.1. Synthesis of PIM-1. PIM-1 was synthesized by polycondensation of TTSBI and TFTPN using the procedure reported elsewhere. ${ }^{15,23-25}$

2.3.2. Synthesis of homopolymer and copolymer. The series of homopolymers and copolymers were synthesized by polycondensation of monomers 4 with TFTPN and monomers 4/TTSBI (different mol ratio) with TFTPN, respectively, using a procedure similar to that of PIM-1. The homopolymers are referred to as PIM-CO4a/b (I to V)-100 (e.g. PIM-CO4a-I-100) and the copolymers are identified as PIM-CO4a/b (I to V)-75, 50, 30 (e.g. PIM-CO4aI-75, PIM-CO4aI-50 and PIM-CO4aI-30), where PIM stands for polymer of intrinsic microporosity, CO4a/b (I to $\mathrm{V}$ ) is the comonomer number and suffixes $-100,-75,-50,-30$ refer to the comonomer/TTSBI ratio in the copolymers.

Polymerization procedure. TTSBI, TFTPN and (or) comonomer were dissolved in DMAc to form a homogeneous solution in a three necked flask equipped with magnetic stirrer and argon inlet. Addition of $\mathrm{K}_{2} \mathrm{CO}_{3}$ (in excess of $1: 1.2$ based on $\mathrm{OH}$ component(s)) caused a solution colour change from yellow to orange. The reaction mixture was immersed in an oil bath preheated at $150{ }^{\circ} \mathrm{C}$ and within minutes the reaction mixture became increasingly viscous, then diethyl benzene (DEB) was added dropwise to maintain stirring and stirring was continued for $30 \mathrm{~min}$. The hot reaction mixture was poured into methanol and the precipitated yellow solid was collected by filtration. The crude polymer was refluxed for $1 \mathrm{~h}$ in deionized water, filtrated and dried under vacuum at $120^{\circ} \mathrm{C}$ for $48 \mathrm{~h}$. The polymer was dissolved in chloroform and reprecipitated in methanol. In case the polymer product was insoluble in chloroform and dichlorobenzene, it was purified by washing with acetone.

\subsection{Membrane preparation}

Thick polymer films were prepared by solution casting method from polymer solution (concentration 1-2\%, w/w) into accurately levelled Teflon circular dish. For low boiling solvent such as chloroform, the dish was covered by a lid and the system was gently purged using a stream of $\mathrm{N}_{2}$. After solvent evaporation, the prepared films were delaminated and conditioned by soaking in methanol for approximately $4 \mathrm{~h}$. The methanol treated membranes were dried in high vacuum for $16 \mathrm{~h}$ at 120 ${ }^{\circ} \mathrm{C}$. The thickness of the membranes was measured by a digital micrometer (Deltascopes MP2C) to 50-75 $\mu \mathrm{m}$. Moreover, thin film composite (TFC) membranes for long term study were prepared on polyacrylonitrile (PAN) microporous support (average pore size of $25 \mathrm{~nm}$ and with $15 \%$ surface porosity) from polymer solution (conc. 0.5 or $1 \% \mathrm{w} / \mathrm{w}$ ) in chloroform by an in house manufactured casting machine.

\subsection{Gas permeation measurement}

Permeabilities of four pure gases $\left(\mathrm{H}_{2}, \mathrm{~N}_{2}, \mathrm{CO}_{2}\right.$, and $\left.\mathrm{CH}_{4}\right)$ were measured by a pressure increase time-lag apparatus at $30{ }^{\circ} \mathrm{C}$. Permeability $(P)$, diffusivity $(D)$, solubility $(S)$ and selectivity $(\alpha)$ for gases A and B were determined under steady state conditions by the following Equation:

$$
\begin{gathered}
P=D S=\frac{V_{\mathrm{p}} l\left(p_{\mathrm{p}^{2}}-p_{\mathrm{p}^{1}}\right)}{A R T \Delta t\left(p_{\mathrm{f}}-\left(p_{\mathrm{p}^{2}}+p_{\mathrm{p}^{1}}\right) / 2\right)} \\
D=\frac{l^{2}}{6 \theta} \\
\alpha_{\mathrm{A} / \mathrm{B}}=\frac{P_{\mathrm{A}}}{P_{\mathrm{B}}}=\frac{D_{\mathrm{A}} S_{\mathrm{A}}}{D_{\mathrm{B}} S_{\mathrm{B}}}
\end{gathered}
$$

$V_{\mathrm{p}}$ is the constant permeate volume, $R$ is the gas constant, $l$ is the film thickness, $A$ is the effective area of membrane, $\Delta t$ is the time for permeate pressure increase from $p_{\mathrm{p}^{1}}$ to $p_{\mathrm{p}^{2}}, p_{\mathrm{f}}$ is the feed pressure, and $\theta$ is the time-lag. The solution-diffusion transport model ${ }^{26-29}$ was applied for discussing the gas transport properties of dense polymer membranes, and the selectivities of membranes for gas " $\mathrm{A}$ " relative to another gas " $\mathrm{B}$ " is the ratio of their permeabilities obtained from eqn (3).

\section{Results and discussions}

\subsection{Monomer synthesis}

The comonomers $[\mathbf{4 a}(\mathbf{I}-\mathbf{V}), \mathbf{4} \mathbf{b}(\mathbf{I}-\mathbf{V})]$ were synthesized using a multistep synthetic route as shown in Scheme 2. The key intermediates for the synthesis of $\mathbf{4 a -}(\mathbf{I}-\mathbf{V}), \mathbf{4 b}(\mathbf{I}-\mathbf{V})$ are $\mathbf{1}(\mathbf{a}-\mathbf{b})$ and were prepared by slight modification to a published procedure, ${ }^{\mathbf{3 0 , 3 1}}$ from the condensation reaction between the appropriate aldehyde (R) and 1,2-dimethoxybenzene in conc. $\mathrm{H}_{2} \mathrm{SO}_{4}$. The advantage of starting with methoxy groups is the enhanced solubility compared to hydroxyl groups and the high stability towards oxidation. The anthracene maleic anhydride derivatives $2(\mathbf{a}-\mathbf{b})$ were prepared in high yield by Diels-Alder reaction between $\mathbf{1}(\mathbf{a}-\mathbf{b})$ and maleic anhydride in toluene using the procedure described by Milton C. Kloetzel. ${ }^{32}$

The imides at the maleic anhydride substituent $[\mathbf{3 a}(\mathbf{I}-\mathbf{V})$, $\mathbf{3 b}(\mathbf{I}-\mathbf{V})]$ were prepared by reacting $\mathbf{2}(\mathbf{a}-\mathbf{b})$ with different aniline substituents $(\mathrm{X})$ in the presence of glacial acetic acid and acetic anhydride for water removal to give the precursors [3a(I-V), $\mathbf{3 b}(\mathbf{I}-\mathbf{V})]$. The original synthesis of the maleimides was described by Leslie R.D. et $a l .{ }^{33}$ Here it is slightly modified so as to allow for the simple introduction of substituted anilines and to provide symmetrical structure. Finally, the tetramethoxy precursors $[\mathbf{3 a}(\mathbf{I}-\mathbf{V}), \mathbf{3} \mathbf{b}(\mathbf{I}-\mathbf{V})]$ were readily demethylated by $\mathrm{BBr}_{3}$ in $\mathrm{CH}_{2} \mathrm{Cl}_{2}$ to give the target tetrahydroxy monomers [4a-(I-V), $\mathbf{4 b}(\mathbf{I}-\mathbf{V})]$. The structures of the monomers were confirmed by FTIR, ${ }^{1} \mathrm{H}$ NMR, ${ }^{13} \mathrm{C}$ NMR, and elemental analysis.

${ }^{1} \mathrm{H}$ NMR spectra of tetrahydroxy monomers $\mathbf{4 a - I}$ and $\mathbf{4 b}-\mathbf{b}$ are shown in Fig. $1 .{ }^{1} \mathrm{H}$ NMR signals corresponding to characteristic aromatic protons of monomer 4a-I are observed at 6.5, 6.6, 6.7 and $7.3 \mathrm{ppm}$, whereas aliphatic and maleic anhydride protons of 4 a-I are observed at 2 and 2.9 ppm, respectively, as shown in Fig. 1i (4a-I). Monomer 4b-I shows the ${ }^{1} \mathrm{H}$ NMR signals for aromatic protons at the same position as $\mathbf{4 a - I}$ due to identical framework. The aliphatic protons of the butyl groups in $4 \mathbf{b}$-I range from 1.6 to $2.6 \mathrm{ppm}$, the characteristic protons originating from the former maleic anhydride shifted down field to 
<smiles>[R]c1c2cc(OC)c(OC)cc2c([R])c2cc(OC)c(OC)cc12</smiles>

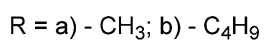<smiles>[X]c1ccccc1</smiles>

I<smiles>Cc1cc(C)cc(I)c1</smiles><smiles>CCOc1ccccc1</smiles><smiles>[R]C12c3cc(OC)c(OC)cc3C([R])(c3cc(OC)c(OC)cc31)C1(C(=O)O)C(=O)OC(=O)C21</smiles>

$2(a, b)$

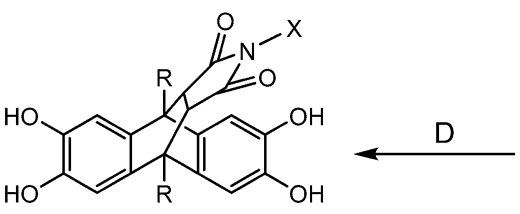

$4 a$ (I to $V)$ $4 b$ (I to V)

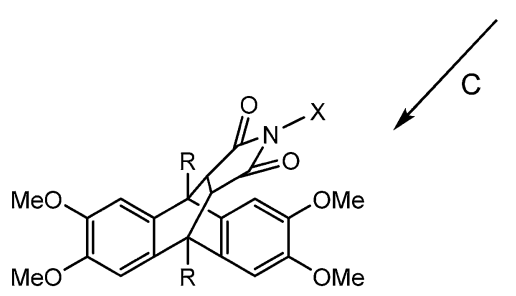

$3 a(I$ to $V)$ $3 b$ (I to $V$ )

Scheme 2 Synthetic route of monomer, reagents and conditions (A) when R: $a=-\mathrm{CH}_{3}$; conc. $\mathrm{H}_{2} \mathrm{SO}_{4}, 18 \mathrm{~h}$, room temperature; when R: $b=$ $-\mathrm{C}_{4} \mathrm{H}_{9}$; acetonitrile, conc. $\mathrm{H}_{2} \mathrm{SO}_{4}$, room temperature, $2 \mathrm{~h}$. (B) Maleic anhydride, toluene, $150{ }^{\circ} \mathrm{C}, 2 \mathrm{~h}$. (C) Acetic anhydride, acetic acid, $130{ }^{\circ} \mathrm{C}, 24 \mathrm{~h}$ (D) $\mathrm{BBr}_{3}, \mathrm{DCM}$, room temperature, $18 \mathrm{~h}$.

$3.3 \mathrm{ppm}$ because of the electron rich butyl groups, as depicted in Fig. 1ii (4b-I).

The other monomer pairs of $\mathbf{4 a - b}$ and the substituted anilines compare similarly in their analytical data. The monomer structures (4a-I and $\mathbf{4 b}$-I) were also confirmed by FT-IR (ESI Fig. S1 $\dagger$ ). In general, the monomers on the basis of $4 \mathbf{a}$ are of low solubility, dissolving only in DMSO, DMF or DMAc, but not in low boiling solvents like chloroform, THF or acetone. The butyl groups, however, improve the solubility of monomers on $\mathbf{4 b}$ basis, allowing dissolution in chloroform or THF.

Generally, PIMs are obtained via polycondensation reaction at different temperatures; the original procedure of Budd/ McKeown ${ }^{12}$ runs at $60{ }^{\circ} \mathrm{C} / 3-4$ days. Guiver et al. ${ }^{34}$ reduced the reaction time to few minutes by heating the reaction mixture to $155{ }^{\circ} \mathrm{C}$ and maintained stirrability by addition of toluene. We adopted the latter procedure to prepare PIM-1 and other PIMs but use diethyl benzene instead of toluene to keep the temperature inside the reaction mixture at $150{ }^{\circ} \mathrm{C}$. The high temperature could cause the problem of a retro Diels-Alder reaction in the new series of monomers and therefore TGA measurements were conducted on $\mathbf{4 a - I}$ and $\mathbf{4 b} \mathbf{b}-\mathbf{I}$ monomers to a thermal ramp up to $700{ }^{\circ} \mathrm{C}$ under argon atmosphere at a rate of $5 \mathrm{~K} \mathrm{~min}^{-1}$. By coupling with FT-IR the evolved gases were analysed. 4a-I proved to be stable at least till $280{ }^{\circ} \mathrm{C}$ and decomposition started at $295{ }^{\circ} \mathrm{C}$. The $1^{\text {st }}$ extract was measured at $334{ }^{\circ} \mathrm{C}$, but a retro Diels-Alder with evaporation of phenylmaleimide was observed only at the $2^{\text {nd }}$ extract at $358^{\circ} \mathrm{C}$. Fig. $2 \mathrm{a}$ shows the TGA mass loss analysis for 4a-I. Inset Fig. 2a shows the intensity of FTIR signals, which is connected to maximum gradient of weight loss in the TGA trace.

Two extractions were carried out at two different temperatures $\left(334{ }^{\circ} \mathrm{C} \& 358^{\circ} \mathrm{C}\right)$. Analysis of first extraction of gaseous decomposition product by FTIR spectra (Fig. 2 at $334^{\circ} \mathrm{C}$ ) shows a band at $2215 \mathrm{~cm}^{-1}-2350 \mathrm{~cm}^{-1}$ and $3015 \mathrm{~cm}^{-1}$ (accompanied with low concentration of phenylmaleimide band also) which fitted to $\mathrm{CO}, \mathrm{CO}_{2}$ and $\mathrm{CH}_{4}$ reference gas spectra from National Institute of Standard and Technology (NIST) Chemistry WebBook $^{35}$ (see ESI for gas spectra of methane in Fig. S2 $\dagger$ ). The second extraction of gaseous decomposition at $358^{\circ} \mathrm{C}$ shows a band at $575 \mathrm{~cm}^{-1}, 1185 \mathrm{~cm}^{-1}, 1360 \mathrm{~cm}^{-1}$ and $1785 \mathrm{~cm}^{-1}$, which fitted to reference gas spectra of phenylmaleimide ${ }^{35}$ (shaded region in inset Fig. 2a). Similarly, the TGA-FTIR measurement of $4 \mathbf{b}-\mathrm{I}$ is depicted in Fig. $2 \mathrm{~b}$. The significant mass loss started at $250^{\circ} \mathrm{C}$ and between $250-375^{\circ} \mathrm{C}$, approximately $60 \mathrm{wt} \%$ loss was observed in the compound, which corresponds to decomposition of $\mathbf{4 b}$-I backbone. Analysis of first and second extraction of gaseous decomposition product by FTIR spectra at $334{ }^{\circ} \mathrm{C}$ and $358{ }^{\circ} \mathrm{C}$ shows a mixture of bands. The signals shows at 576 $\mathrm{cm}^{-1}, 1184 \mathrm{~cm}^{-1}, 1361 \mathrm{~cm}^{-1}, 1786 \mathrm{~cm}^{-1}$ (for phenylmaleimideshaded region inset Fig. 2(a and b)), 2215-2410 $\mathrm{cm}^{-1}$ (for $\mathrm{CO}_{2}$ ) and $3015 \mathrm{~cm}^{-1}$ (for $\mathrm{C}_{4} \mathrm{H}_{10}$ ), which fitted with the reference gas spectra of respective molecules (see ESI for gas spectra of butane Fig. S2 $\dagger$ ). ${ }^{35}$ In difference to $4 a-I$ the retro Diels-Alder occurred already at lower temperature, in the $1^{\text {st }}$ extract, 


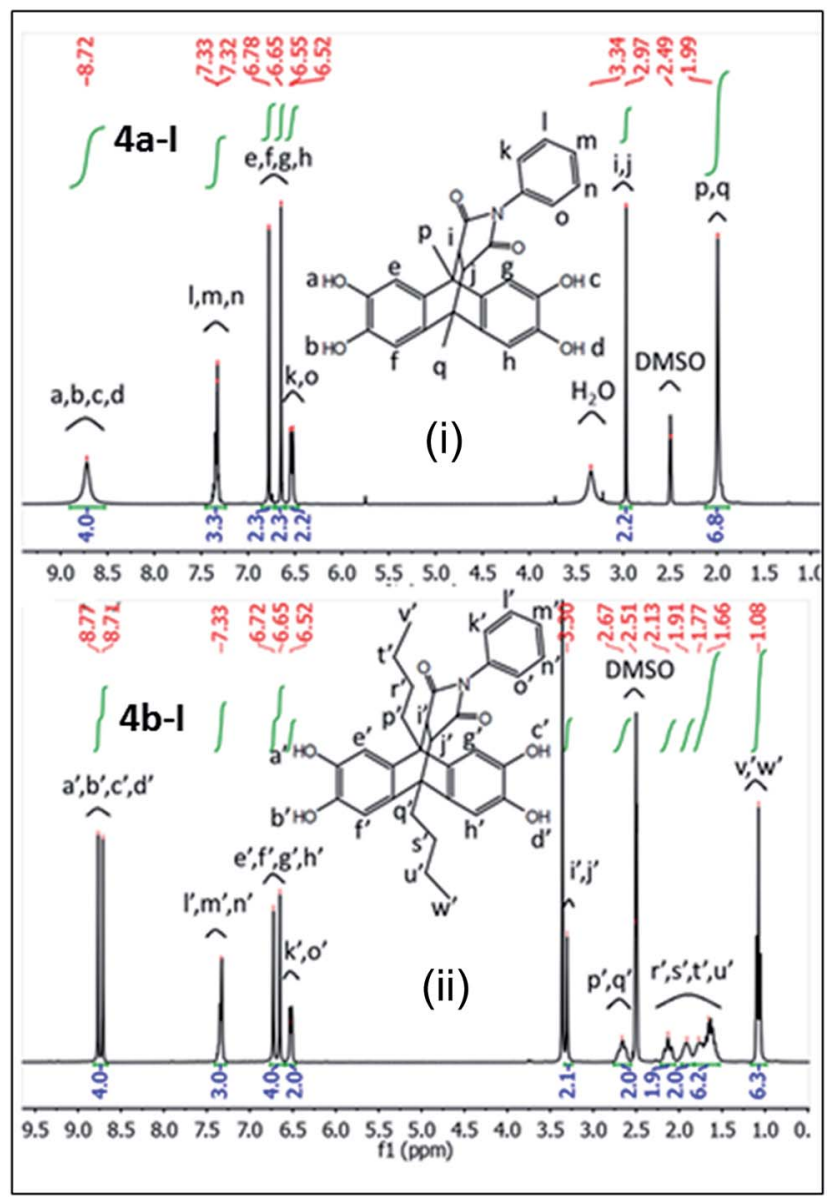

Fig. $1{ }^{1} \mathrm{H}-\mathrm{NMR}$ spectra of (i) $4 a-1$ and (ii) $4 \mathrm{~b}-\mathrm{I}$.

however, stability till $250{ }^{\circ} \mathrm{C}$ was established. Therefore, the polymerisation at high temperature remains unaffected. The three dimensional (3D) view of TGA-FTIR of $4 \mathbf{4 a - I}$ and $\mathbf{4 b}$-I are shown in ESI Fig. S3 and S4.†

\subsection{Polymerization}

The series of homopolymers and copolymers from the various monomers were synthesized via polycondensation reaction by reacting tetrahydroxy monomers with equimolar amounts of TFTPN, catalyzed by an excess of $\mathrm{K}_{2} \mathrm{CO}_{3}$, using a modified procedure similar to the synthesis of PIM-1 (Scheme 3). ${ }^{15,23-25,36}$

According to the mechanism of polycondensation reaction of poly(arylene ether)s, high temperature and high monomer concentration are favourable for increasing solubility of phenoxide salt and growing the polymer chain, hence, the occurrence of cyclic oligomers and crosslinked structure can be effectively reduced. In the present work, a slightly modified polymerization procedure ${ }^{15}$ was used compared to previously reported polymerization reaction..$^{34}$ DEB was used in polymerization reaction instead of toluene (as described in procedure), which is advantageous not only to provide solubility enhancement of polymer but also provides a high boiling point solvent to the reaction. ${ }^{15,25}$ To keep the reaction mixture less viscous, additional amounts of DEB were added. The presence of DEB required precipitation of the polymers in methanol. The basic features of reaction and polymer characterization such as

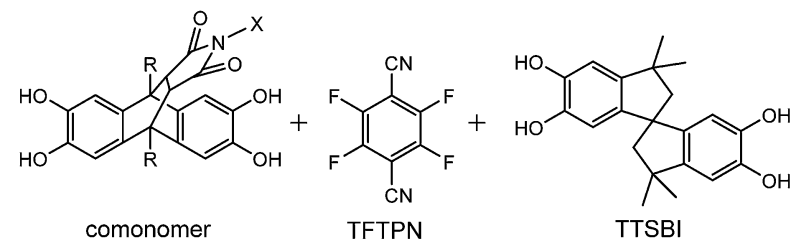<smiles>[In][Tl]</smiles>

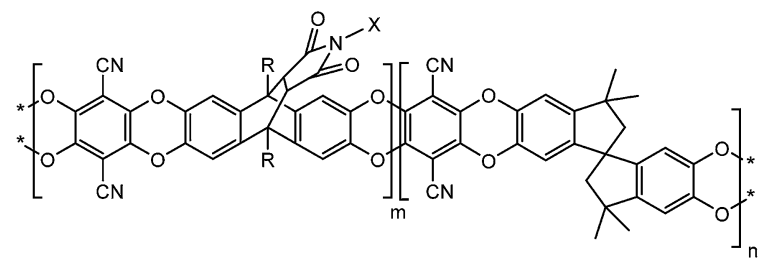

Homopolymer $4 \mathrm{a}(\mathrm{I}$ to $\mathrm{V})-100$ and $4 \mathrm{~b}(\mathrm{I}-\mathrm{V})-100$ (i.e.; $\mathrm{m}: \mathrm{n}=1: 0)$ Copolymer $4 a(I$ to $V)-30,50,75$ and $4 b(I$ to $V)-30,50,75$ (i.e.; $m: n=3: 1,1: 1,1: 2.3$ )

Scheme 3 Synthesis of homopolymer and copolymers, 1-reagent and condition, $\mathrm{K}_{2} \mathrm{CO}_{3}, \mathrm{DMAC}$ (dimethylacetamide), DEB (diethylbenzene), $150{ }^{\circ} \mathrm{C}, 1 / 2 \mathrm{~h}$
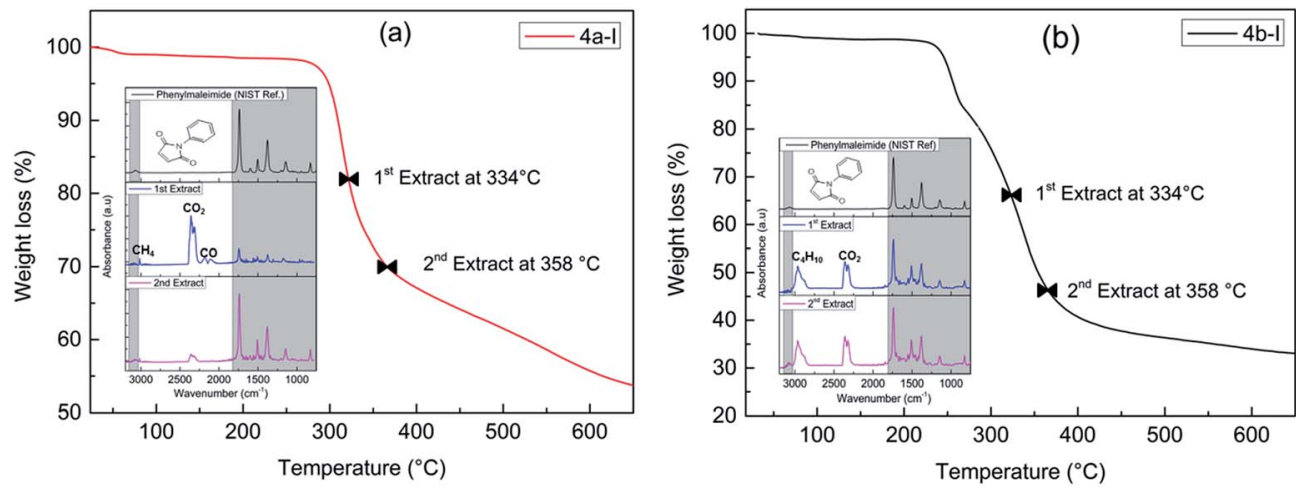

Fig. 2 TGA coupled with FTIR spectra of (a) $4 a-1$ and (b) $4 b-1$. 
composition, molecular weight, thermal properties and copolymer composition estimated by ${ }^{1} \mathrm{H}-\mathrm{NMR}$ are listed in Table 1.

The average molar masses and polydispersities of homopolymers and copolymers were determined by SEC using polystyrene standards. For copolymers with methyl and butyl groups on the bridge head position [4a and $4 \mathbf{b}$ (I to V)] reasonable molecular weights with rather high polydispersity indices were obtained, which is consistent with the results of a typical polycondensation reaction. Table 1 also shows the copolymers composition, estimated from ${ }^{1} \mathrm{H}-\mathrm{NMR}$ spectra by integration of the peaks in the aromatic region (at $\sim 6.4 \mathrm{ppm}$ ) for TTSBI monomer (PIM-1 monomer) and aliphatic region for comonomer $\mathbf{4 a}$ and $\mathbf{4 b}$ (at $\sim 3.5 \mathrm{ppm}$ ). In each case the calculated composition reflected closely to the comonomer composition of the reaction (as an example shown in Fig. 3). Furthermore, the comparison of FT-IR spectra of copolymers shows a distinguishable increase of the $\mathrm{C}=\mathrm{O}$ stretching vibration between $1660-1730 \mathrm{~cm}^{-1}$ with increasing content of comonomer 4a-I and 4b-I (ESI Fig. S5 and S6†). Differential scanning calorimetry (DSC) of all polymers showed no evidence of glass transition below the degradation temperature.

Moreover, the homopolymers [PIM-CO4b(I-V)-100] derived from the new imido-monomers and TFTPN were synthesized under the same reaction conditions as PIM-1 (i.e. $150{ }^{\circ} \mathrm{C}$ and 30 $\min )^{34}$ and gave sufficiently high molecular weights. As the homopolymers obtained from monomers $\mathbf{4 a}(\mathbf{I}-\mathbf{V})$ are insoluble in most organic solvents, the molecular weight determination by SEC was not possible. However, the homopolymers obtained from monomers $\mathbf{4 b}$ (I-V) exhibited good solubility in many organic solvents, e.g. chloroform and THF, due to the butyl groups on bridge head position, allowing both mechanically strong and flexible films to be cast. As an example, the images of homopolymer films derived from $\mathbf{4 b}$ (I-V) are shown in ESI Fig. S7. $\dagger$ Furthermore, thermo-gravimetric analysis (TGA) of homopolymers and copolymers derived from $\mathbf{4 a}$ (I-V) series showed that the onset of decomposition temperature is quite similar, while TGA of $\mathbf{4} \mathbf{b}$ (I-V) polymers and copolymers series showed two different onset decomposition temperatures (see

Table 1 Physical properties of PIM-1, homopolymers and copolymers derived from the monomer $4 \mathrm{a}$ (I to V) and $4 \mathrm{~b}$ (I to V)

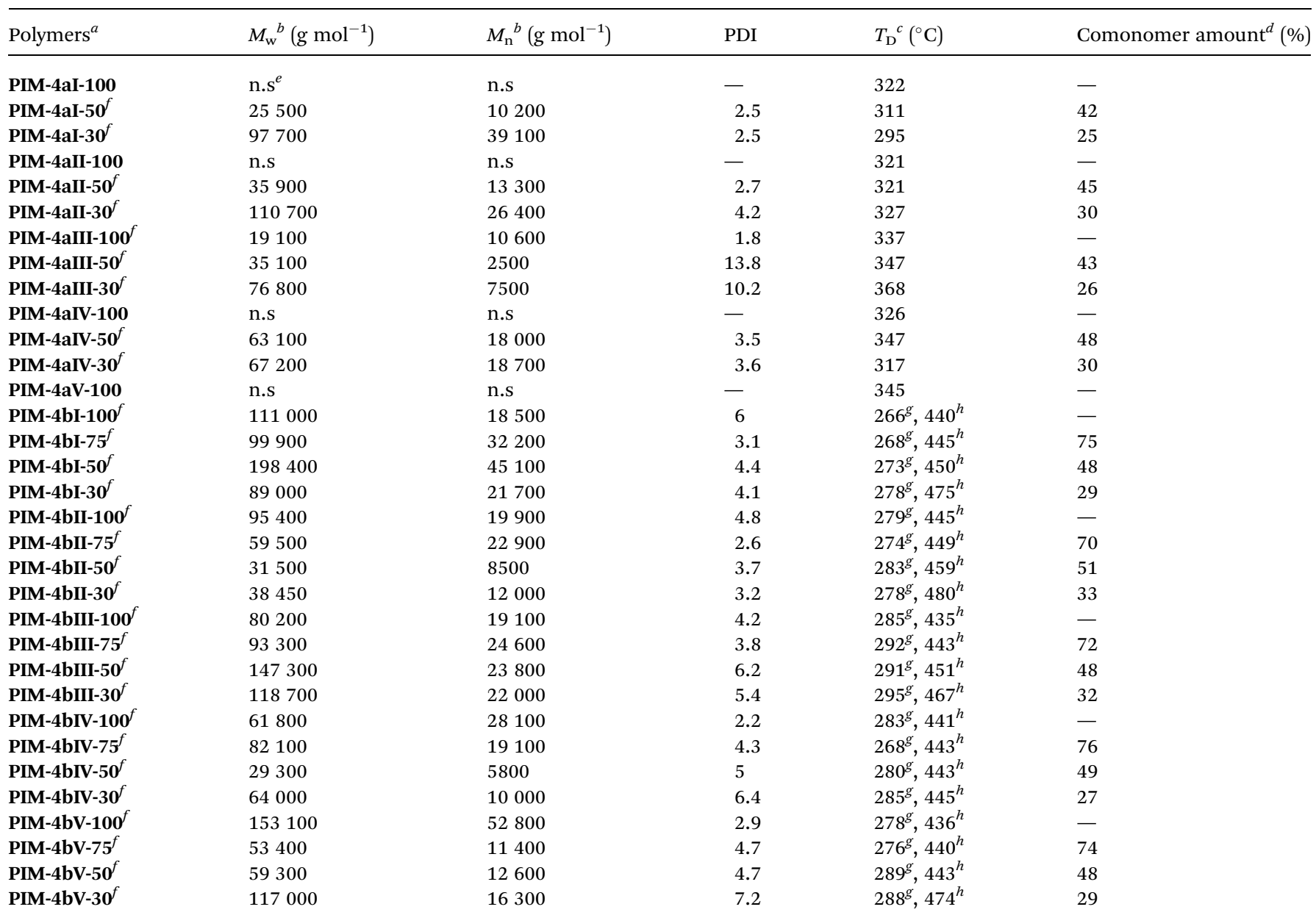

${ }^{a}$ Each polymer is defined by the comonomer(s) from which they were made. For copolymers, the percentage of the contribution of comonomers to the TTSBI monomer (PIM-1 monomer) composition is given at the end of each polymer name. ${ }^{b}$ From SEC analysis with calibrated polystyrene standards. ${ }^{c}$ Actual onset temperature of decomposition. ${ }^{d}$ Anthracene maleimide commoner-estimated by ${ }^{1} \mathrm{H}-\mathrm{NMR}$. ${ }^{e}$ n.s means that the polymer is insoluble in an appropriate solvent for SEC $\left(\right.$ i.e. $\left.\mathrm{CHCl}_{3}\right) .{ }^{f}$ Soluble in $\mathrm{CHCl}_{3}$ and THF. ${ }^{g}$ Primary onset temperature of decomposition.

${ }^{h}$ Secondary onset temperature of decomposition. 

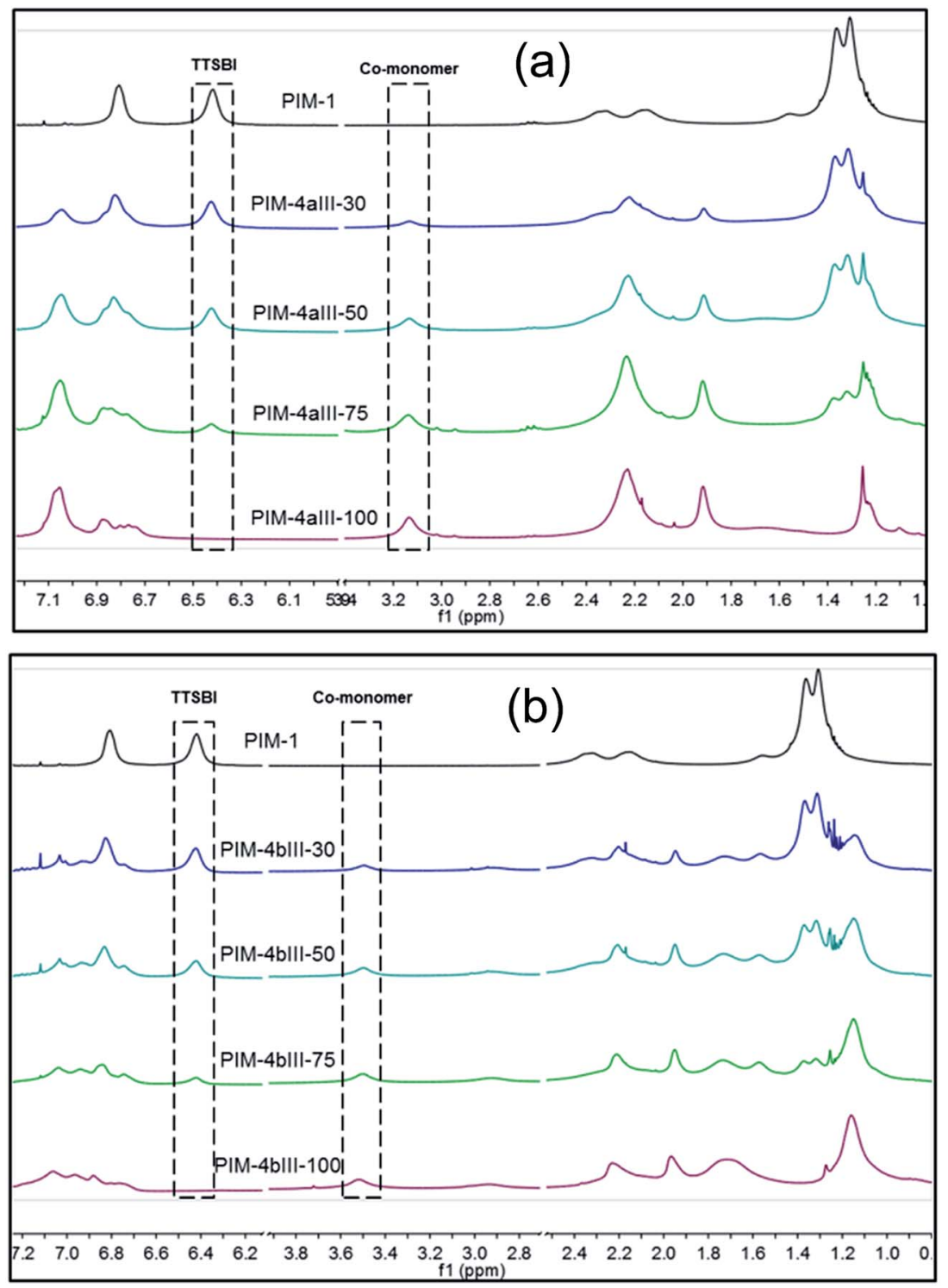

Fig. 3 Comparative ${ }^{1} \mathrm{H}-\mathrm{NMR}$ spectra of copolymer with different composition derived from the comonomer (a) $4 \mathrm{a}-\mathrm{III}$ and (b) $4 \mathrm{~b}$-III.

ESI Fig. S8 $\dagger$ ). In case of $\mathbf{4 b}(\mathbf{I}-\mathbf{V})$, the initial decomposition corresponds to decomposition of alkyl group along with maleimide derivatives and the second decomposition caused by breaking the backbone chain of the polymer.

We observed that the surface area of homopolymer (i.e. without any spiro unit) derived from the monomer [4a and $\mathbf{4 b}$ (I-V)] were in the range of $520-660 \mathrm{~m}^{2} \mathrm{~g}^{-1}$. The reason could be a greater cohesive interaction between the polymer chains due to the polar imido group, or occlusion of maleimide derivatives in the microporosity. The copolymers derived from the monomer (4b-III) were selected for further study. Table 2 gives BET surface area from nitrogen adsorption for copolymers with different ratio of monomer (4b-III) and also for the PIM-1 prepared under identical conditions in this study.

Fig. 4a compares the nitrogen adsorption/desorption isotherm at liquid nitrogen temperature (77 K) for PIM-4bIII50 and PIM-1. Both polymers show high uptake at low relative pressure, a characteristic of microporous materials as defined by IUPAC (pore size $<2 \mathrm{~nm}$ ). ${ }^{10}$ Both materials also demonstrated hysteresis, the desorption curve lying above the adsorption curve down to relative low pressures, a typical behaviour of microporous polymers that may be attributed to polymer swelling effects $\left(p / p_{0}<0.2\right) .{ }^{14}$ Analysis of the very low relative pressure regions of the adsorption isotherm by the HorvathKawazoe (HK) method gives the pore width distribution shown in Fig. 4 b. Both polymers show a broad distribution of effective pore size in the micropore region (0.6-0.65 $\mathrm{nm})$.

From the literature, ${ }^{14}$ it was assumed that the roof-shaped structure once embedded within the polymer network possesses greater rigidity, which helps to enhance the microporosity. Comparing the triptycenecontaining A-B monomer precursor, it seems that the additional substituents on the maleimide unit make the polymer even more rigid but also fill some of the space created by the rigid polymer frameworks. 
Table 2 BET surface area of PIM-1 and the copolymers with (4bIII)

\begin{tabular}{|c|c|c|}
\hline Polymers & $\begin{array}{l}\text { Ratio (spiro } \\
\text { unit:4bIII) }\end{array}$ & $\begin{array}{l}\text { Surface area } \\
\left(\mathrm{m}^{2} \mathrm{~g}^{-1}\right)\end{array}$ \\
\hline PIM-1 $^{a}$ & $100: 0$ & 780 \\
\hline PIM-1 ${ }^{b}$ & $100: 0$ & 830 \\
\hline PIM-4bIII-25 & $75: 25$ & 850 \\
\hline PIM-4bIII-50 & $50: 50$ & 870 \\
\hline PIM-4bIII-75 & $25: 75$ & 790 \\
\hline PIM-4bIII-100 & $0: 100$ & 660 \\
\hline
\end{tabular}

\subsection{Gas separation performance}

Free standing membranes of polymers for gas separation could be prepared by solution-casting and they were tough enough to allow measurement of gas permeability. Pure gas permeability coefficients $\left(P_{\mathrm{i}}\right)$ for $\mathrm{H}_{2}, \mathrm{~N}_{2}, \mathrm{O}_{2}, \mathrm{CO}_{2}$, and $\mathrm{CH}_{4}$ were measured at $30{ }^{\circ} \mathrm{C}$ by time-lag instrument on dense polymer films of homopolymers [PIM-CO4b-100] and series of copolymers derived from monomers $\mathbf{4 b}(\mathbf{I}-\mathbf{V})$. Gas permeation measurement was not possible for the homopolymer and copolymers obtained from the monomer $\mathbf{4 a}(\mathbf{I}-\mathbf{V})$ because of their low molar masses leading to poor film forming properties. Permeabilities and ideal permselectivities $\left(\alpha_{\mathrm{ij}}=P_{\mathrm{i}} / P_{\mathrm{j}}\right)$ are shown in ESI Tables $\mathrm{S} 1$ and $\mathrm{S} 2, \dagger$ respectively. All data were obtained from methanol treated membranes as mentioned in the experimental part. Immersing the membranes in methanol reverses the prior film formation history, in a manner similar to protocols previously developed for microporous polyacetylenes and PIM-1. ${ }^{38,39}$ For comparison, PIM-1 data of this present work and from literature are also included. ${ }^{40}$

It is seen that the structure of anthracene maleimide derivatives exerts a strong effect on the observed permeability and permselectivity. Most homopolymers and the series of copolymers exhibit slightly higher selectivity, coupled with a reduction in gas permeability, compared to PIM-1. An exception is the series of copolymers of $\mathbf{4 b}$-III, as they show comparable or slightly higher permeabilities than PIM-1 (shaded circle in Fig. 5).
For all other series of copolymer-PIMs (CO-PIMs), gas permeabilities were observed to increase with increasing ratios of TTSBI and therefore increasing similarity to PIM-1. PIMCO4bIII-100 has higher $\mathrm{CH}_{4}$ and $\mathrm{N}_{2}$ permeability as PIM-1 and also the other copolymers with $\mathbf{4 b}$-III behave similar to PIM-1. A reason for this should be the substitution of the aniline in $o^{-}$ position preventing rotation of the imido-group and consequently a close packing of the polymer chains. Also, the highly rigid three dimensional structures of anthracene maleimides 4a-III and 4b-III can be concluded on the ${ }^{1} \mathrm{H}-\mathrm{NMR}$ spectra showing three single peaks for the aromatic methyl groups at aniline. Another reason for comparable permeabilities could be the similar pore size distribution and higher surface area of copolymer 4b-III, shown in Table 2. For the other CO-PIMs, the $p$-substituted aniline is free in rotation and could be fitted in free volumes within the chain more easily leading to a reduction of permeability but increasing the selectivity.

The overall gas separation performances of newly synthesized polymers with comparison to Robeson's upper bound (1991 and 2008) for $\mathrm{O}_{2} / \mathrm{N}_{2}, \mathrm{CO}_{2} / \mathrm{CH}_{4}$ and $\mathrm{CO}_{2} / \mathrm{N}_{2}$ gas pairs are shown in Fig. 6.

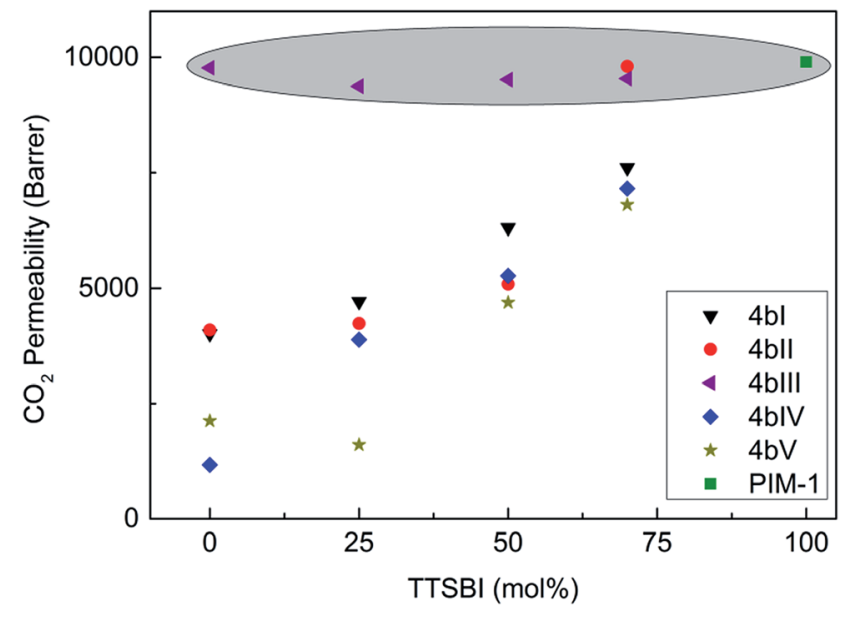

Fig. $5 \mathrm{CO}_{2}$ permeability of copolymers derived from monomer $4 \mathrm{~b}$ (I to $\mathrm{V}$ ) as a function of TTSBI content (mol\%).
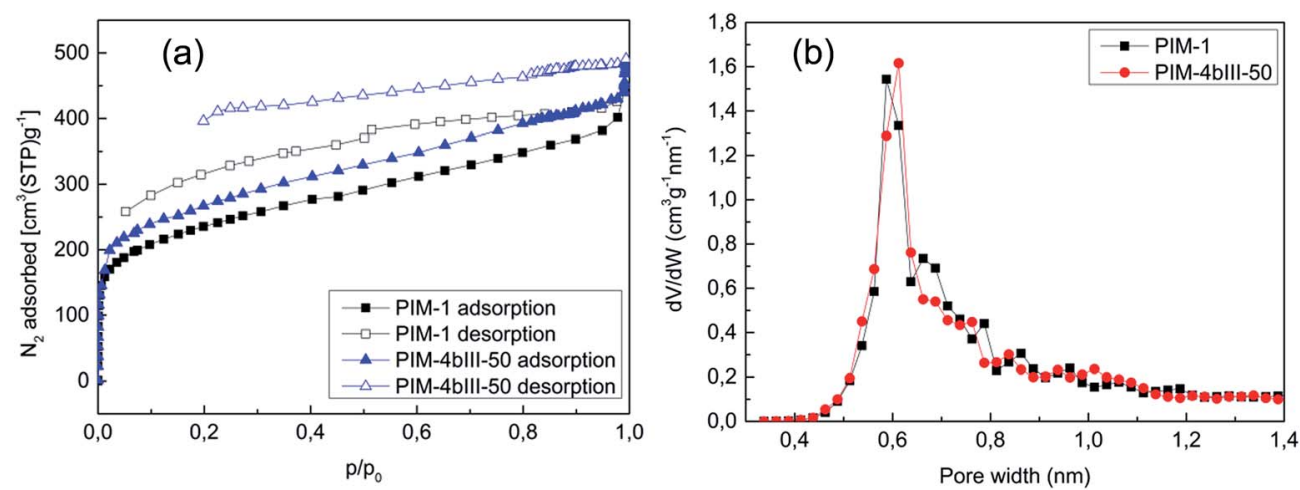

Fig. 4 (a) $\mathrm{N}_{2}$ adsorption (filled symbols) and desorption (open symbols) isotherm at $77 \mathrm{~K}$ for PIM-1 and PIM-4blll-50 and (b) pore width distribution obtained by analysis of $\mathrm{N}_{2}$ adsorption at $77 \mathrm{~K}$ by the Horvath-Kawazoe (HK) method. 
The performance of CO-PIMs for the gas pairs $\mathrm{O}_{2} / \mathrm{N}_{2}$ falls below the 2008 Robeson's upper bound. But for $\mathrm{CO}_{2} / \mathrm{CH}_{4}$ and $\mathrm{CO}_{2} / \mathrm{N}_{2}$ gas pairs, most of CO-PIMs show values near to upper bound 2008. However, for these gas pairs the selectivity is mainly due to the higher solubility dependent rather than diffusivity of $\mathrm{CO}_{2}$. Moreover, the polymers derived from newly synthesized monomers exhibit the competing behaviour to the promising spirobifluorene based PIM (SBF-PIM), ${ }^{41}$ thermally rearranged (TR) polymers ${ }^{42}$ polymers and tetrazole substituted PIMs (TZ-PIMs), ${ }^{43}$ that lie above the 2008 upper bound for $\mathrm{CO}_{2} /$ $\mathrm{CH}_{4}$ and $\mathrm{CO}_{2} / \mathrm{N}_{2}$. Furthermore, the gas separation performance of CO-PIMs with comparison to upper bound (1991 and 2008) for other gas pairs such as $\mathrm{H}_{2} / \mathrm{CH}_{4}, \mathrm{H}_{2} / \mathrm{CO}_{2}$ and $\mathrm{H}_{2} / \mathrm{N}_{2}$ are shown in ESI Fig. S9. $\dagger$

From an application point of view, temperature effects were studied on one of these copolymer membranes at higher temperature. Fig. 7 shows the permeability of $\mathrm{N}_{2}, \mathrm{CH}_{4}$ and $\mathrm{CO}_{2}$ for PIM-1 and PIM-CO4bIII copolymer membranes as a function of the inverse absolute temperature (temperature range 283-343 $\mathrm{K}$, single gas at 1 bar feed pressure). Fig. 7 depicts that the permeability of the $\mathrm{N}_{2}$ and $\mathrm{CH}_{4}$ increased with increasing in temperature while for $\mathrm{CO}_{2}$ the permeability decreased with increasing temperature.
In order to understand the temperature dependence of $\mathrm{N}_{2}$, $\mathrm{CH}_{4}$, and $\mathrm{CO}_{2}$ permeabilities of these membranes, resulting data were correlated with the Arrhenius equation. Thus, the activation energy of permeation $\left(E_{\mathrm{p}}\right)$ was determined by the Arrhenius relationship:

$$
P=P_{0} \exp \left(\frac{-E_{\mathrm{p}}}{R T}\right)
$$

$P$ is permeability, $P_{0}$ is a pre-exponential factor, $E_{\mathrm{p}}$ is the activation energy of permeation ( $\left.\mathrm{J} \mathrm{mol}^{-1}\right), R$ is the gas constant $\left(8.314 \mathrm{~J}\left(\mathrm{~mol}^{-1} \mathrm{~K}^{-1}\right)\right)$ and $T$ is the absolute temperature.

The activation energies of permeation $\left(E_{\mathrm{p}}\right)$ determined from the slope of the Arrhenius plot are shown in Table 3. It provides a qualitative measure of free volume and the size of molecular gaps between chain segments. ${ }^{44}$ In general, the activation energy of permeation is the sum of the activation energy of diffusion $\left(E_{\mathrm{d}}\right)$ and the enthalpy of sorption $\left(\Delta H_{\mathrm{s}}\right)$,

$$
E_{\mathrm{p}}=E_{\mathrm{d}}+\Delta H_{\mathrm{s}}
$$

The activation energy of diffusion is a size-related parameter, its value depends on the size of the penetrant, the intra- and
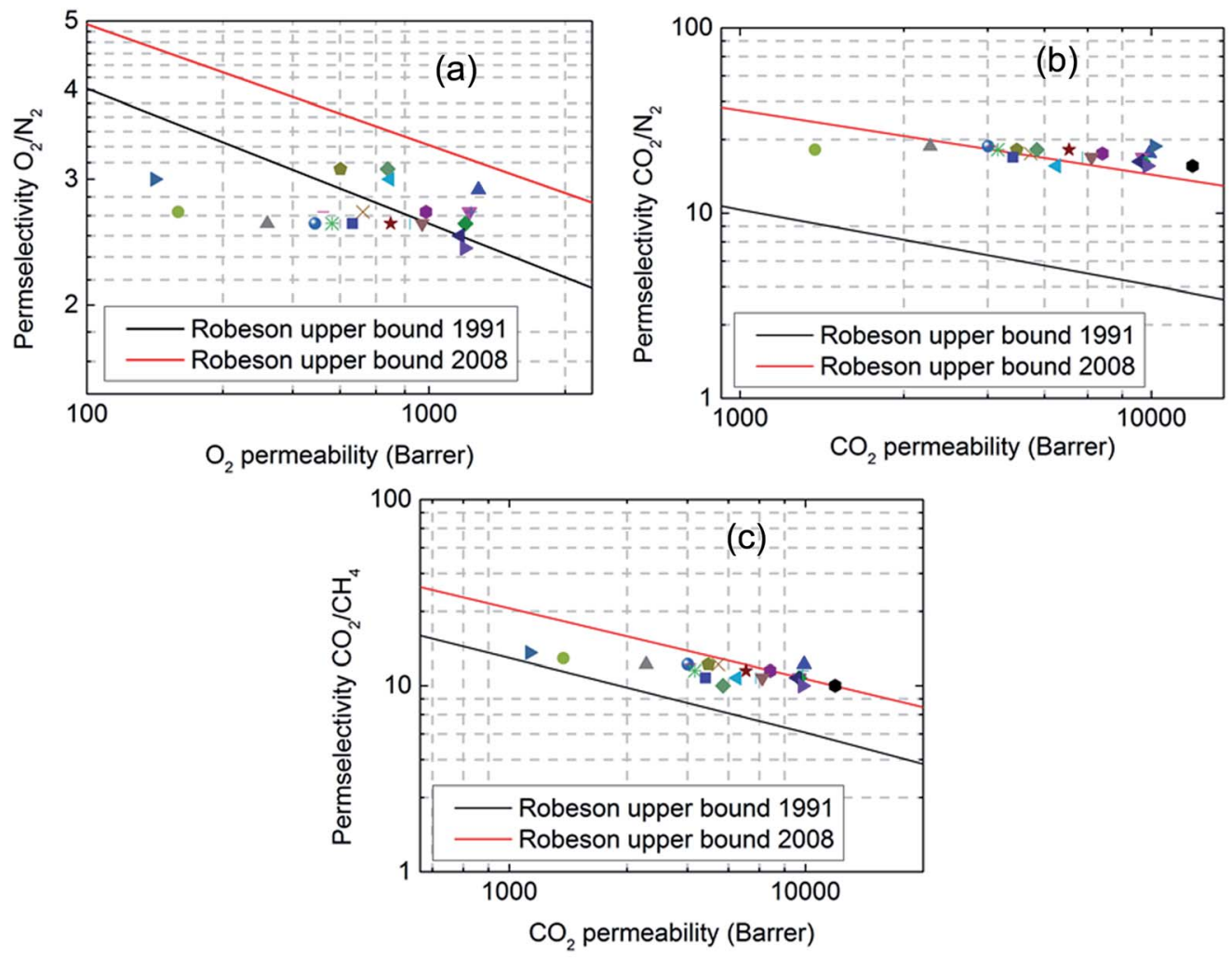

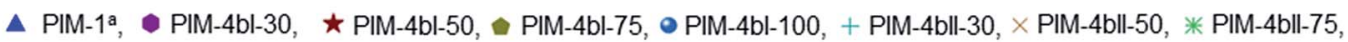

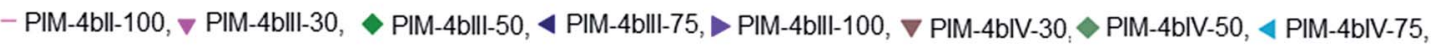

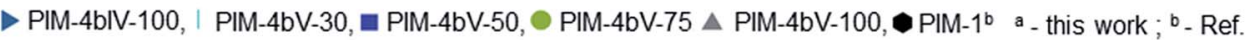

Fig. 6 Robeson plot for (a) $\mathrm{O}_{2} / \mathrm{N}_{2}$ (b) $\mathrm{CO}_{2} / \mathrm{CH}_{4}$ and (c) $\mathrm{CO}_{2} / \mathrm{N}_{2}$ gas pairs showing data for methanol treated PIM-1 and CO-PIMs. The black and red lines represent the 1991 and 2008 upper bound, respectively. 


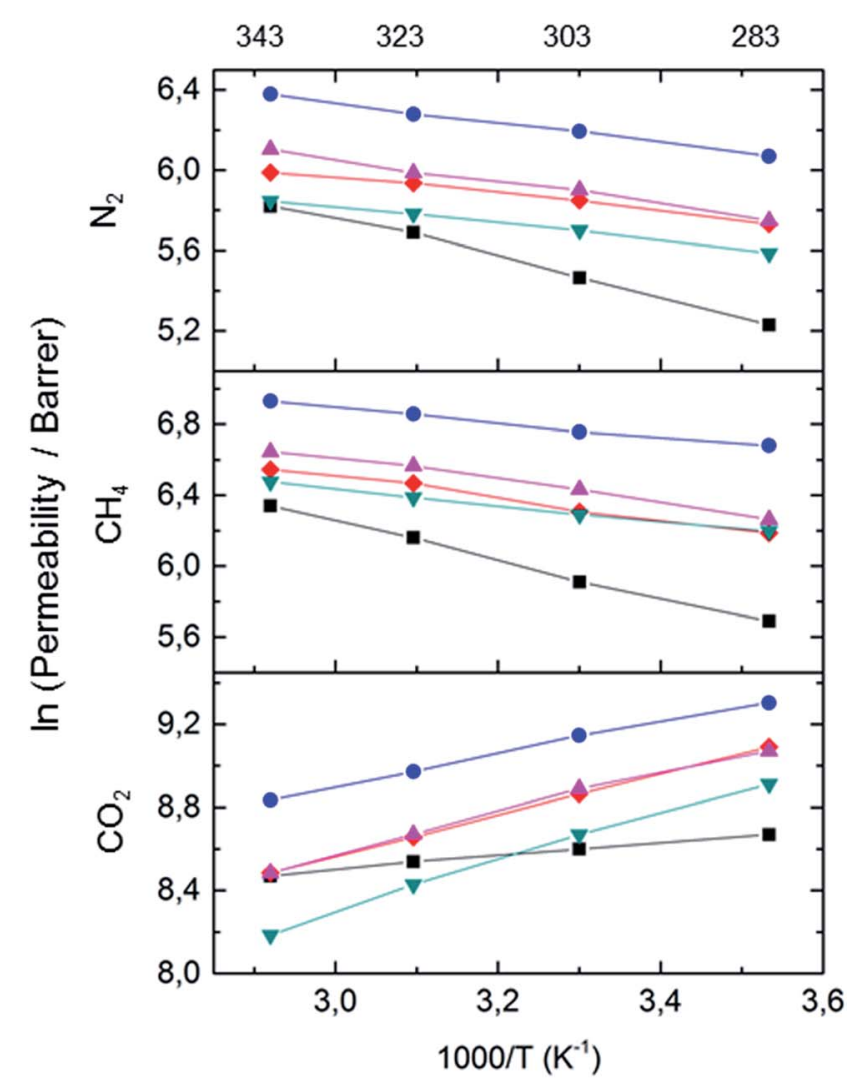

Fig. 7 Permeability of $\mathrm{N}_{2}, \mathrm{CH}_{4}$ and $\mathrm{CO}_{2}$ in PIM-1 and copolymers derived from the comonomer $(4 \mathrm{~b}-\mathrm{I})$ as a function of reciprocal temperature (๑-PIM-4bIII-100, $\Delta$-PIM-4bIII-75, -PIM-4bIII-50, $\boldsymbol{\nabla}$-PIM-4bIII-30, $\mathbf{\square}$-PIM-1).

inter-molecular gaps between polymer chains, and the chain mobility. For activated diffusion processes, $E_{\mathrm{d}}$ is positive, meaning that diffusion coefficients increase with increasing temperature. The heat of sorption is related to the thermodynamic interaction of the penetrant with the polymer. Sorption of gases in polymers is typically an exothermic process. Thus, solubility of gases in polymers decreases with increasing temperature, and $H_{\mathrm{s}}$ is negative.

From Fig. 7, it was observed that gas permeability of $\mathrm{N}_{2}$ and $\mathrm{CH}_{4}$ for these membranes increases with increasing temperature, because $E_{\mathrm{d}}+H_{\mathrm{s}}>0$ or $\left|E_{\mathrm{d}}\right| /\left|H_{\mathrm{s}}\right|>1$, a typical behaviour of

Table 3 Activation energy of permeation of PIM-1 and copolymer obtained from the monomer $4 \mathrm{~b}$-III

\begin{tabular}{llrl}
\hline & \multicolumn{2}{l}{$E_{\mathrm{p}}\left(\mathrm{kJ} \mathrm{mol}^{-1}\right)$} & \\
\cline { 2 - 4 } Membranes & $\mathrm{N}_{2}$ & $\mathrm{CH}_{4}$ & $\mathrm{CO}_{2}$ \\
\hline PIM-4bIII-100 & 3.5 & 3.4 & -9.8 \\
PIM-4bIII-75 & 4.1 & 3.8 & -6.4 \\
PIM-4bIII-50 & 4.7 & 5.2 & -8.2 \\
PIM-4bIII-30 & 3.5 & 5.0 & -8.0 \\
PIM-1 & 8.1 & 11.9 & -2.7
\end{tabular}

conventional glassy polymers. An exception to this rule is the temperature dependence of gas permeability in high-freevolume PIM-1 and its copolymer for condensable gases such as $\mathrm{CO}_{2}$, because gas permeabilities decrease with increased temperature (i.e. $\left|E_{\mathrm{d}}\right| /\left|H_{\mathrm{s}}\right|<1$ ). This indicates that the effect of highly sorbed gases like $\mathrm{CO}_{2}$ does not affect the permeation rate of lighter gases in subsequent runs. The negative activation energies of permeation in high free volume glassy polymer result from very small activation energies of diffusion. ${ }^{45}$ Furthermore, negative activation energies of permeation are routinely observed for microporous solids in which the pore dimensions are relatively large in comparison with the diffusing gas molecules. ${ }^{44}$ On the other hand $\mathrm{CH}_{4}$ and $\mathrm{N}_{2}$ permeabilities of PIM-1 depend strongly on temperature and their $E_{\mathrm{p}}$ values are essentially of the same order of magnitude as those of conventional glassy polymers.

The long term stability tests for gases such as $\mathrm{O}_{2}, \mathrm{~N}_{2}$ and $\mathrm{CO}_{2}$ were also carried out for two copolymers compositions derived from the monomer 4b-III (i.e. PIM-4b-III-50, PIM-4b-III-100). The copolymer was cast to thin film composite membranes on a PAN support and during the $800 \mathrm{~h}$ of the test these membranes were continuously flushed with $\mathrm{N}_{2}$ at 2 bar pressure. $\mathrm{N}_{2}, \mathrm{O}_{2}$ and $\mathrm{CO}_{2}$ permeances were measured at random over a period of 800 h. It was observed that the prepared copolymer composite membrane does not show any significant improvement in terms of permeance and selectivity $\mathrm{O}_{2} / \mathrm{N}_{2}$ and $\mathrm{CO}_{2} / \mathrm{N}_{2}$ gas pairs and behaves almost similar to PIM-1.

\section{Conclusion}

Ten new tetrahydroxy anthracene based monomers with imidoaniline substitution (4a,b I to $\mathbf{~}$ ) were synthesized, characterized and employed in polycondensation reactions with TFTPN to form homopolymers and copolymers in combination with TTSBI. All synthetic procedures are easy, efficient and suitable for scale-up. The achieved PIMs were characterized by NMR, FTIR, TGA, SEC and partly by BET surface area. The polymers obtained from monomers (4a) show low solubility in suited solvents such as $\mathrm{CHCl}_{3}$ and THF compared to polymers obtained from the monomer (4b). Changing the alkyl group (R) at the bridged anthracene from methyl to $n$-butyl group enhanced significantly the solubility. Therefore, polymers from monomer (4b) series could be cast into membranes and measured in single gas permeation of $\mathrm{N}_{2}, \mathrm{O}_{2}, \mathrm{CO}_{2}$ and $\mathrm{CH}_{4}$. Permeability of the new polymers is mostly lower or comparable to PIM-1, while selectivities are slightly higher or comparable to PIM-1. These results offer a variety of possible new monomers and polymer structures with potential in microporous materials for application in membrane technology and other areas where soft matters with large intrinsic free volume combined with chemical functionality are required.

\section{Acknowledgements}

The authors would like to thank Silke Dargel and Petra Merten for technical support. This work was financially supported by 
Helmholtz Association of German Research Centres through the Helmholtz Portfolio MEM-BRAIN.

\section{Notes and references}

1 M. P. Tsyurupa and V. A. Davankov, React. Funct. Polym., 2002, 53, 193-203.

2 B. S. Ghanem, K. J. Msayib, N. B. McKeown, K. D. M. Harris, Z. Pan, P. M. Budd, A. Butler, J. Selbie, D. Book and A. Walton, Chem. Commun., 2007, 0, 67-69.

3 N. B. McKeown, S. Hanif, K. Msayib, C. E. Tattershall and P. M. Budd, Chem. Commun., 2002, 0, 2782-2783.

4 C. D. Wood, B. Tan, A. Trewin, H. Niu, D. Bradshaw, M. J. Rosseinsky, Y. Z. Khimyak, N. L. Campbell, R. Kirk, E. Stöckel and A. I. Cooper, Chem. Mater., 2007, 19, 20342048.

5 T. Masuda, E. Isobe, T. Higashimura and K. Takada, J. Am. Chem. Soc., 1983, 105, 7473-7474.

6 K. Nagai, T. Masuda, T. Nakagawa, B. D. Freeman and I. Pinnau, Prog. Polym. Sci., 2001, 26, 721-798.

7 K. Tanaka, M. Okano, H. Toshino, H. Kita and K.-I. Okamoto, J. Polym. Sci., Part B: Polym. Phys., 1992, 30, 907-914.

8 A. Y. Alentiev, V. P. Shantarovich, T. C. Merkel, V. I. Bondar, B. D. Freeman and Y. P. Yampolskii, Macromolecules, 2002, 35, 9513-9522.

9 N. B. McKeown and P. M. Budd, Chem. Soc. Rev., 2006, 35, 675-683.

10 N. B. McKeown and P. M. Budd, Macromolecules, 2010, 43, 5163-5176.

11 M. Carta, K. J. Msayib, P. M. Budd and N. B. McKeown, Org. Lett., 2008, 10, 2641-2643.

12 P. M. Budd, B. S. Ghanem, S. Makhseed, N. B. McKeown, K. J. Msayib and C. E. Tattershall, Chem. Commun., 2004, 230-231.

13 D. Fritsch, P. Merten, K. Heinrich, M. Lazar and M. Priske, J. Membr. Sci., 2012, 401-402, 222-231.

14 T. Emmler, K. Heinrich, D. Fritsch, P. M. Budd, N. Chaukura, D. Ehlers, K. Rätzke and F. Faupel, Macromolecules, 2010, 43, 6075-6084.

15 D. Fritsch, G. Bengtson, M. Carta and N. B. McKeown, Macromol. Chem. Phys., 2011, 212, 1137-1146.

16 T. Defize, R. Riva, C. Jérôme and M. Alexandre, Macromol. Chem. Phys., 2012, 213, 187-197.

17 M. Helm, M. Petermeier, B. Ge, R. Fiammengo and A. Jäschke, J. Am. Chem. Soc., 2005, 127, 10492-10493.

18 M. Chandra and S. K. Silverman, J. Am. Chem. Soc., 2008, 130, 2936-2937.

19 K. T. Gagnon, S.-Y. Ju, M. B. Goshe, E. S. Maxwell and S. Franzen, Nucleic Acids Res., 2009, 37, 3074-3082.

20 H. Durmaz, A. Dag, A. Hizal, G. Hizal and U. Tunca, J. Polym. Sci., Part A: Polym. Chem., 2008, 46, 7091-7100.

21 Z. Shi, J. Luo, S. Huang, Y.-J. Cheng, T.-D. Kim, B. M. Polishak, X.-H. Zhou, Y. Tian, S.-H. Jang, J. D. B. Knorr, R. M. Overney, T. R. Younkin and A. K. Y. Jen, Macromolecules, 2009, 42, 2438-2445.
22 X. Liu and J. K. Snyder, J. Org. Chem., 2008, 73, 2935-2938. 23 M. M. Khan, V. Filiz, G. Bengtson, M. M. Rahman, S. Shishatskiy and V. Abetz, Procedia Eng., 2012, 44, 18991901.

24 M. Khan, V. Filiz, G. Bengtson, S. Shishatskiy, M. Rahman and V. Abetz, Nanoscale Res. Lett., 2012, 7, 504.

25 M. M. Khan, V. Filiz, G. Bengtson, S. Shishatskiy, M. M. Rahman, J. Lillepaerg and V. Abetz, J. Membr. Sci., 2013, 436, 109-120.

26 J. G. Wijmans and R. W. Baker, J. Membr. Sci., 1995, 107, 121.

27 A. M. Shishatskii, Y. P. Yampol'skii and K. V. Peinemann, J. Membr. Sci., 1996, 112, 275-285.

28 M. M. Rahman, V. Filiz, S. Shishatskiy, S. Neumann, M. M. Khan and V. Abetz, Procedia Eng., 2012, 44, 1523-1526.

29 M. M. Rahman, V. Filiz, S. Shishatskiy, C. Abetz, S. Neumann, S. Bolmer, M. M. Khan and V. Abetz, J. Membr. Sci., 2013, 437, 286-297.

30 A. Müller, M. Raltschewa and M. Papp, Ber. Dtsch. Chem. Ges. A, 1942, 75, 692-703.

31 Y. V. Shklyaev and Y. V. Nifontov, Russ. Chem. Bull., 2002, 51, 844-849.

32 M. C. Kloetzel, in Organic Reactions, Wiley, 2011, pp. 1-59.

33 L. R. Dix, J. R. Ebdon, N. J. Flint, P. Hodge and R. O'Dell, Eur. Polym. J., 1995, 31, 647-652.

34 N. Du, J. Song, G. P. Robertson, I. Pinnau and M. D. Guiver, Macromol. Rapid Commun., 2008, 29, 783-788.

35 U.S. Department of Commerce.

36 M. M. Khan, G. Bengtson, S. Shishatskiy, B. N. Gacal, M. Mushfequr Rahman, S. Neumann, V. Filiz and V. Abetz, Eur. Polym. J., 2013, 49, 4157-4166.

37 P. M. Budd, B. S. Ghanem, S. Makhseed, N. B. McKeown, K. J. Msayib and C. E. Tattershall, Chem. Commun., 2004, 0, 230-231.

38 A. J. Hill, S. J. Pas, T. J. Bastow, M. I. Burgar, K. Nagai, L. G. Toy and B. D. Freeman, J. Membr. Sci., 2004, 243, 37-44.

39 K. Nagai, L. G. Toy, B. D. Freeman, M. Teraguchi, T. Masuda and I. Pinnau, J. Polym. Sci., Part B: Polym. Phys., 2000, 38, 1474-1484.

40 P. M. Budd, N. B. McKeown, B. S. Ghanem, K. J. Msayib, D. Fritsch, L. Starannikova, N. Belov, O. Sanfirova, Y. Yampolskii and V. Shantarovich, J. Membr. Sci., 2008, 325, 851-860.

41 C. G. Bezzu, M. Carta, A. Tonkins, J. C. Jansen, P. Bernardo, F. Bazzarelli and N. B. McKeown, Adv. Mater., 2012, 24, 59305933.

42 H. B. Park, C. H. Jung, Y. M. Lee, A. J. Hill, S. J. Pas, S. T. Mudie, E. Van Wagner, B. D. Freeman and D. J. Cookson, Science, 2007, 318, 254-258.

43 N. Du, H. B. Park, G. P. Robertson, M. M. Dal-Cin, T. Visser, L. Scoles and M. D. Guiver, Nat. Mater., 2011, 10, 372-375.

44 I. Pinnau and L. G. Toy, J. Membr. Sci., 1996, 109, 125-133. 45 T. Masuda, Y. Iguchi, B.-Z. Tang and T. Higashimura, Polymer, 1988, 29, 2041-2049. 